\title{
Centrosomal Enrichment and Proteasomal Degradation of SYS-1/ $\beta$-catenin Requires the Microtubule Motor Dynein
}

3

\section{Abstract}

The $C$. elegans Wnt/ $\beta$-catenin Asymmetry (WBA) pathway utilizes asymmetric regulation of SYS$1 / \beta$-catenin and POP-1/TCF coactivators. W $\beta$ A differentially regulates gene expression during cell fate decisions, specifically by asymmetric localization of determinants in mother cells to produce daughters biased towards their appropriate cell fate. Despite the induction of asymmetry, $\beta$-catenin localizes symmetrically to mitotic centrosomes in both mammals and $C$. elegans. Due to the mitosis-specific localization of SYS-1 to centrosomes and enrichment of SYS-1 at kinetochore microtubules when SYS-1 centrosomal loading is disrupted, we investigated active trafficking in SYS-1 centrosomal localization. Here, we demonstrate that trafficking by microtubule motor dynein is required to maintain SYS-1 centrosomal enrichment, by dynein RNAi-mediated decreases in SYS-1 centrosomal enrichment and by temperature-sensitive allele of the dynein heavy chain. Conversely, we observe depletion of microtubules by Nocodazole treatment or RNAi of dynein-proteasome adapter ECPS-1 exhibits increased centrosomal enrichment of SYS-1. Moreover, disruptions to SYS-1 or negative regulator microtubule trafficking are sufficient to significantly exacerbate SYS-1 dependent cell fate misspecifications. We propose a model whereby retrograde microtubule-mediated trafficking enables SYS-1 enrichment at centrosomes, enhancing its eventual proteasomal degradation. These studies support the link between centrosomal localization and enhancement of proteasomal degradation, particularly for proteins not generally considered 'centrosomal'.

\section{Keywords}

Development, Wnt, $\beta$-catenin, microtubule, ECM29, dynein, proteasome, trafficking, elegans, asymmetry, cell cycle, robustness 

pathways to appropriately specify and populate tissues. This is of further importance in the developing embryo, where cell division rapidly alternates between S-phase and mitosis while simultaneously specifying tissues and entire germ layers (Pintard and Bowerman 2019). As such, the cell's response to these signals and the subsequent establishment of asymmetry often begins before asymmetric daughter cells are even 'born' via an asymmetric cell division (ACD) (Sunchu and Cabernard 2020; Juanes, 2020; Fuchs and Chen, 2013; Gómez-López, Petrisch et al 2014). Among the signaling pathways utilized in development is Wnt/ $\beta$-catenin signaling, wherein the binding of Wnt ligands disrupts $\beta$-catenin degradation via dismantling an intracellular destruction complex. This effectively stabilizes $\beta$-catenin, enabling it to activate target genes with transcriptional activator TCF/LEF (Nusse and Clevers 2017). Wnt/ $\beta$-catenin signaling is specialized in its ability to regulate both polarity and proliferation - enabling

42 developing organisms to orient and expand tissues simultaneously for appropriate size and morphology

43 (Loh et al 2016). Due to its role in both proliferation and polarity, Wnt/ $\beta$-catenin signaling is readily adaptable to the adult organism throughout development.

Wnt/ $\beta$-catenin is particularly well adapted to the organization of a patterned stem cell niche for proliferative tissue maintenance (Pinto, Clevers et al 2003; Reilein, Kalderon et al 2017). However, this

47 function in adult tissue homeostasis also associates deleterious dysregulation of Wnt/ $\beta$-catenin signaling with disease states. Throughout transduction of the Wnt signal are proteins vulnerable to oncogenic mutation (Yang, yen 2011; Zhunussova, Djansugurova et al 2019; Liu, Thibodeau et al 2000). However,

50 these often affect their tumorigenicity via dysregulation of the pathway effector $\beta$-catenin, which can 51 promote proliferation via over-and mis-expression of targets like Cyclin D1 and cMyc (Niehrs, Acebron 52 2012; He, Kinzler et al 1998; Shtutman, Ben-Ze'ev et al 1999; Tetsu and McCormick, 1999). Moreover, $\beta$ - 
catenin is a bifunctional protein in mammalian systems. That is, it is distributed between the previously

54 described signaling role while also maintaining cell-cell adhesion at adherens junctions (Niehrs, Acebron 2012; Reya, Clevers 2005), where it facilitates the interaction between membrane protein E-cadherin and the cytoskeleton. The combination of these roles for $\beta$-catenin makes it an attractive target for

57 studying tumorigenesis, both due to hyperproliferative phenotypes and ease of metastasis. However, the dual functions can also lead to confounding pleiotropic phenotypes after manipulation of $\beta$-catenin activity (Reya, Clevers 2005; Tejpar, Alman et al 1999; Geyer, Reis-Filho et al 2011). the adhesive and signaling roles across its four $\beta$-catenins (Sawa, 2012). Here we focus specifically on the $C$. elegans $\beta$-catenin SYS-1 for its role as a transcriptional co-activator in a modified Wnt/ $\beta$-cat signaling pathway, the Wnt/ $\beta$-catenin Asymmetry pathway(W $\beta A$ ) (Phillips, Kimble et al 2007). The W $\beta A$ pathway is a specialized application of the more basic Wnt signaling paradigm, wherein regulation of the co-activator SYS-1 ( $\beta$-catenin) modulates the activity of transcription factor POP-1 (TCF), during asymmetric cell division (Baldwin and Phillips 2018, Lam and Phillips 2017). Mother cells undergoing a

67 W $\beta A$-driven division polarize components of the Wnt signaling pathway along the axis of the division distributing positive regulators of a Wnt response, the Frizzled receptor and downstream effector Disheveled, to the signaled pole of the cell, and negative regulators, members of the $\beta$-Catenin 70 destruction complex APC and Axin, to the unsignaled pole (Baldwin and Phillips 2014; Baldwin, Phillips et 71 al 2016; Sugioka, Sawa et al 2011; Mizumoto and Sawa, 2007). Daughter cells in such a division

72 therefore inherit Wnt signaling components that bias them toward their appropriate cell fate. Contrary 73 to its known role asymmetrically activating Wnt target genes, SYS-1 (and indeed, mammalian $\beta$-catenin 74 (Mbom, Barth et al 2014; Vora, Phillips et al 2020)), unexpectedly localizes symmetrically to mother cell 75 mitotic centrosomes (Phillips, Kimble et al 2007; Huang, Lin et al 2007; Vora and Phillips 2015). 
Previous work has demonstrated that mother cell centrosomal localization of SYS-1 limits SYS-1

77 levels in daughter cells in a proteasome dependent manner (Vora and Phillips 2015). Differential localization of the proteasome during the cell cycle (Amsterdam, Baumeister et al 1993), as well as interactor-specific localization of the proteasome for intracellular structuring (Baldin, Coux et al 2008), suggest that proteasome regulation, localization, and activity are interdependent. Proteasomal subunits have specifically been shown to co-sediment with centrosomal proteins and perinuclear inclusions,

82 indicative of centrosomal localization (Wigley, Thomas et al 1999; Wojcik, DeMartino 2003). Similarly,

83 turnover of the SYS-1 mobile fraction at mitotic centrosomes is enhanced by RPT-4 proteasomal activity

84 (Vora and Phillips 2015). When the localization of SYS-1 to centrosomes is reduced by rsa-2(RNAi), resultant daughter cells retain $40 \%$ additional SYS-1 protein. Perhaps, then, the centrosome is a region where SYS-1 regulatory interactions are concentrated to coordinate its retention and effect on cell fate 87 during mitosis. implied that a more active localization mechanism might be at work, as compared to a passive diffusioncapture mechanism. SYS-1 motility is most directly demonstrated in that the cell maintains a proteasome-dependent, $100 \%$ mobile population of centrosomal SYS-1, which is recycled within 120 seconds (Vora and Phillips 2015). Cells appear to maintain robust localization of SYS-1 to the mitotic centrosome, while the interphase centrosome remains undetectable via SYS-1 fluorescence (Vora and Phillips 2015). However, the mechanism of such temporally specific SYS-1 centrosomal localization is unknown. Several observations indicate that cells utilize the microtubule cytoskeletal network for rapid

96 and directional regulation of specific cargo to precise locales, including: that dynein conditionally traffics

97 transcription factors due to neuronal injury or signaling status, (Ben-Yaakov, Fainzilber et al 2012; 
sufficient to prevent pathogenic accumulation of RNA binding proteins (Deshiumaru, Tsuboi et al 2021). Therefore, we hypothesized that the role of the mitotic centrosome as an MTOC could facilitate the rapid transport of SYS-1 to the pericentriolar material specifically in dividing cells.

The rapid and cell cycle phase-specific enrichment of SYS-1 at the centrosome suggests that it may also depend on active transport. Further, accumulation at a highly active MTOC like the mitotic centrosome is most likely accomplished by the microtubule minus end-directed dynein motor complex (Vora and Phillips 2015; Priyanga, Bhaka-Guha et al 2021). The dynein motor is a multi-subunit complex composed of a pair of ATP-ase, microtubule-binding heavy chains responsible for the force-generating steps of the motor, and a variable complement of smaller light, intermediate, and accessory chains that alter the cargo binding or processivity of the motor (Reck-Peterson, Carter et al 2018; Jha and Surrey 2015). The dynein motor is multifunctional - it provides pulling forces, positions organelles, and can mediate rapid and conditional movement of proteins and RNAs (Carminati and Stearns 1997; Woźniak, Allan et al 2009; Harada, Hirokawa et al 1998; Hanz, Fainzilber et al 2003; Bullock and Ish-Horowicz 2001). For example, injured rat axons have demonstrated a dynein intermediate chain-dependent nuclear localization and coimmunoprecipitation of the transcription factor STAT3, in order to appropriately adopt an injury-dependent transcriptional response (Ben-Yaakov, Fainzilber et al 2012). Dynein specifically is the motor complex most often directed towards the MTOC and is known to be active during mitosis (Jha and Surrey 2015). Therefore, we investigated dynein-dependent trafficking as an ideal mechanism for SYS-1 localization to the MTOC mitotic centrosomes.

Here we demonstrate that microtubules and minus-end microtubule-mediated trafficking are required for the centrosomal recruitment of both SYS-1 and the negative regulators responsible for its regulation there. We evaluate the extent to which SYS-1 utilizes dynein-mediated trafficking to accumulate at centrosomes. Using both long- and short-term disruptions to dynein and microtubulemediated trafficking to evaluate SYS-1 localization and SYS-1-dependent cellular activity up to and 
124 including cell fate changes, we demonstrate that SYS-1 and its negative regulators both require

125 microtubule-mediated trafficking for appropriate regulation of SYS-1. Together, these data characterize

126 a system for enhancing the robustness of the W $\beta A$ signaling pathway by maintaining consistent

127 interaction of SYS- 1 with negative regulators at the mitotic centrosome.

128 Results

SYS-1 Overexpression and Centrosomal Uncoupling Increases SYS-1 Localization to Regions of Dynein

Enrichment

131 To test the role of microtubule-mediated trafficking in SYS-1 centrosomal localization, we investigated

132 what trafficking mechanisms are necessary for SYS-1 regulation. We compared fluorescently tagged

133 SYS-1 protein localization to that of MT trafficking components. Of most interest was the cytoplasmic

134 dynein motor complex, since its components have been shown to localize to mitotic centrosomes,

135 kinetochore microtubules, and cortically across species (Schmidt, Van den Heuvel et al 2017; Tame,

136 Medema et al 2014). The dynein localization pattern corresponded to that of GFP::SYS-1 to cortical and

137 microtubule dense regions. Moreover, kinetochore and cortical localization was more common upon

138 centrosomal uncoupling, complete loss of visible enrichment of centrosomal SYS-1, by RNAi depletion of

139 the spindle assembly and scaffolding protein RSA-2(Fig. 1A).

While GFP::SYS-1 has been observed at mitotic centrosomes in many dividing cells throughout

141 development, localization to these cortical and kinetochore 'secondary' sites is uncommon

142 (accumulation at either or both of these sites occurring in $34.5 \%$ of embryos) in untreated embryos but

143 the frequency of this localization approximately doubles, to 69.1\%, with rsa-2(RNAi) (Fig.1A). Uncoupling

144 SYS-1 protein from the centrosome by rsa-2(RNAi), thereby decreasing processing of the protein, allows

145 us to more frequently observe SYS-1 at sites of dynein localization (Vora and Phillips 2015, Vora and

146 Phillips 2017)(Fig.1A). Localization to kinetochore microtubules, cortex, and the MTOC centrosome 
therefore suggests that SYS-1 may be a cargo of the comparably localized dynein and kinesin microtubule motors (Ferenz, Wadsworth et al 2010; Schmidt, Van den Heuvel et al 2017).

\section{Knockdown of Dynein Complex Subunits Reduces SYS-1 Centrosomal Localization}

Given the observation that SYS-1 localizes to cortical and kinetochore sites that also exhibit enrichment for microtubule motors, we investigated a functional role for microtubule-mediated trafficking in SYS-1 localization. We began by performing an RNAi screen against various components of the dynein motor complex and associated proteins, eventually expanding outward to additional motors and microtubule-affecting proteins (Fig. S1). Specifically, by RNAi depletion of these microtubule trafficking proteins, we assayed the centrosomal enrichment of GFP::SYS-1. We expected depletion of transport proteins that play a role in SYS-1 centrosomal transport to be defective in the maintenance of SYS-1 centrosomal enrichment when SYS-1 centrosomal clearance was otherwise unaffected. In order to evaluate the centrosome-specific enrichment of SYS-1, we considered two ways of comparing the extent of fluorescently-tagged SYS-1 localization to centrosomes: 1) the centrosomal enrichment index (CEI)(similar to that described in Gao, Smith et al 2017), the centrosomal fluorescence intensity adjusted by subtraction of the background cytoplasmic fluorescent intensity of the surrounding cell, to control for backgrounds that may pleiotropically influence SYS-1 expression, and 2) the SYS-1 CEI evaluated as a ratio to the same enrichment factor of representative Peri Centriolar Material (PCM) factor $\gamma$-tubulin (Tubulin Enrichment Index, or TEI), to avoid false positives via defects in PCM maturation (CEI/TEI) (Fig. 1B- $\left.\mathrm{B}^{\prime \prime}\right)$. We then compared these centrosomal enrichment metrics to those seen in established rsa2(RNAi) centrosomal uncoupling.

As expected, depletion of several dynein subunit knockdowns presented pleiotropic and deleterious effects, including oogenesis and early patterning defects which precluded our SYS-1 
localization assay. We therefore treated animals with partial RNAi knockdowns by commencing RNAi

171 feeding 12-24 hours before imaging as necessary, based on the phenotypic severity. While no

172 treatment disrupted SYS-1 centrosomal levels as severely as rsa-2(RNAi), we did observe several

173 statistically significant decreases in centrosomal SYS-1 enrichment (Fig. 2C - C', Fig. S1). Furthermore,

174 while the total population of some treatments displayed only an intermediate mean loss of SYS-1

175 enrichment, the distribution of SYS-1 CEI values observed in dynein subunit knockdowns extends from

176 wild-type to an rsa-2(RNAi)-like disruption (e.g., Fig. 2 Supplement 1). Dynein knockdown-induced

177 decreases in SYS-1 CEI or CEI/TEI are masked somewhat in the population average by their broad range

178 in phenotype and/or RNAi penetrance (Fig. 1C-C', Fig S2). Most notably, dlc-1(RNAi) and dylt-1(RNAi)

179 each exhibit a significant reduction in centrosomal SYS-1 localization disproportionately larger than the

180 effect on $\gamma$-Tubulin. Loss of centrosomal SYS-1 enrichment was specifically more visible in a transgene

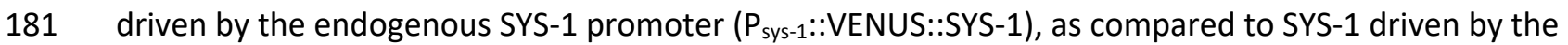

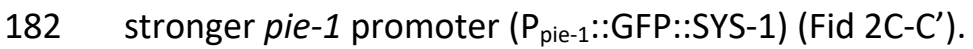

While we observed dynein-dependent decreases in SYS-1 localization, this could be because of

changes in the RSA-2 centrosomal scaffold, which is responsible for capturing SYS-1 at mitotic

centrosomes (Vora and Phillips 2015, Schlaitz, Hyman et al 2007). While some dynein subunits did show slight decreases in centrosomal GFP::RSA-2 - 16\% reduction in dlc-1(RNAi) embryos and $14 \%$ in $d y / t$ 1(RNAi) embryos. Embryos depleted of DLC-1 by RNAi exhibit double this effect on SYS-1 localization, with 39\% and $42 \%$ reductions in Psys-1::VENUS::SYS-1 CEI and CEI/TEI, respectively. DYLT-1 depletion exhibited a similar 14\% decrease in CEI alone, but a 39\% decrease in CEI/TEI. Both knockdowns suggest that tagged SYS-1 is disproportionately removed from centrosomes in dynein knockdowns (Fig. S3)

These data suggest that dynein does have a role in the localization of SYS-1 to centrosomes, but redundancy between components of the dynein motor or between the dynein motor and other 
often severely deleterious, variability of RNAi efficacy may have resulted in incomplete depletion of SYSsuperseded effects on SYS-1 localization, leading to the evaluation of only partial knockdowns. In order to address these issues, we investigated the role of the dynein motor in SYS-1 localization at a more precise temporal resolution via conditional dynein disruption.

\section{Temporally Restricted Dynein Loss of Function Reveals Dynein Requirement for Centrosomal SYS-1}

Accumulation.

201

202

While our screen showed that dynein knockdown results in significant reduction of centrosomal SYS-1, with rare individuals exhibiting $r s a-2$ (RNAi)-like loss of centrosomal SYS-1 accumulation, we were unable to completely recreate a population exhibiting consistently severe disruption to SYS-1 CEI. To distinguish the variety in effect on SYS-1 localization effects between functional redundancies limiting the effects of dynein depletion and the confounding deleterious effects described above, we utilized a temperature sensitive allele of the cytoplasmic dynein heavy chain, dhc-1(or195ts), to conditionally inactivate the cytoplasmic dynein heavy chain. This allele, identified in the Bowerman lab, introduces a lesion to the microtubule binding stalk of DHC-1. This lesion renders the motor slightly hypomorphic at the permissive $15^{\circ} \mathrm{C}$ (with $91 \%$ of embryos remaining viable and fertile) but undergo complete mitotic spindle collapse as little as 30 seconds upon upshift, suggesting that DHC-1 motor function has been nearly completely ablated (O'rourke, Bowerman et al. 2007).

Therefore, we expected that combining the $d h c-1($ ts) allele with fluorescently tagged SYS-1 would allow us to examine a more penetrant dynein knockdown. Notably, this allowed us to overcome both redundancy between dynein accessory chains in SYS-1 trafficking as well as pleiotropic effects of dynein depletion that preclude the observation of mitotic centrosomal SYS-1 (Fig. 2A). As described above, we compared the CEI of $\mathrm{P}_{\text {sys-1 }}:$ VENUS::SYS-1 (VENUS::SYS-1) to that of mCherry:: $\gamma$-Tubulin (TEI). 
217 SYS-1 and $\gamma$-Tubulin are both recruited to centrosomes at a much higher rate during mitosis than in 218 interphase (Vora and Phillips 2015; Raynaud-Messina and Merdes 2007). Because dhc-1(ts) limits the 219 extent of $\gamma$-Tubulin recruitment (Fig. 2B-C), evaluating SYS-1 CEI as a ratio to g-Tubulin TEI ensures that 220 decreases to the SYS-1 CEI must be disproportionately large to be numerically distinct. Consistent with 221 the published hypomorphic nature of the allele at the permissive temperature (O'rourke, Bowerman et 222 al 2007), imaging $d h c-1$ (ts) embryos, shows notable decrease in the SYS-1 CEI of embryos kept at the 223 permissive temperature (Fig. 2 B-D). Following 30-45 seconds of temperature upshift, the SYS-1 224 localization hypomorphy is enhanced, severely reducing SYS-1 centrosomal enrichment (Fig. 2B-C).

225 While there was a decrease in centrosome size, presumably due to roles for dynein in centrosome maturation (Priyanga, Bhakta-Guha et al 2021), we accounted for this by assessing SYS-1 CEI as a ratio to the enrichment of $\gamma$-Tubulin TEI. We observed a CEI/TEI decrease corresponding to decreased centrosomal SYS-1 accumulation, proportionally greater than the reduction in $\gamma$-tubulin (Fig. 2D').

229 Combined with the individual dynein subunit knockdown, the more penetrant phenotype of $d h c-1$ (ts) mutants indicates that SYS-1 requires the dynein microtubule motor complex to localize appropriately to centrosomes. Additionally, it seems likely that redundancy of peripheral motor components or

232 incomplete knockdown is responsible for the variability within individual subunit depletions seen above

233 (Fig 1C-C').

MT depolymerization experiments reveal that SYS-1 centrosomal dynamics are affected both

236 To determine the extent to which microtubule-based trafficking in general is responsible for SYS-1

237 centrosomal localization, we treated gravid adults and their embryos with $>100 \mu \mathrm{M}$ Nocodazole, a

238 microtubule-destabilizing agent. This dose, while sufficient for a rapid acute response in adult animals, 239 either caused no effect on fertilized embryos because of the eggshell or prevented the production of 
additional embryos via germline arrest (data not shown). We therefore turned to a lower dose, longerterm Nocodazole treatment: 12 hours at $25 \mu \mathrm{g} / \mathrm{mL}(83 \mu \mathrm{M})$. Maternal Nocodazole dosing allowed us to obtain embryos with limited microtubule networks, but sufficient spindle development to enter mitosis (Fig. 3A). Surprisingly, when treated with nocodazole, the centrosomal enrichment of the brighter $\mathrm{P}_{\text {pie- }}$ 1::GFP::SYS-1 (GFP::SYS-1) increased after Nocodazole treatment (Fig. 3B, C). was not because of off-target effects, we compared maternal feeding to that of permeabilized embryos. At 10.5 $\mu \mathrm{M}$ these embryos formed sufficient microtubule networks to enter mitosis and form spindles, but these spindles were often inappropriately rotated or positioned ( $61 \%$ of imaged embryos, $n=11 / 18$ ), suggesting that astral microtubules have been severely disrupted (Fig. S4A). We observed a similar CEI increase in GFP::SYS-1 in these embryos (Fig. S4B). This is consistent with a model where both SYS-1 recruitment to centrosomes and SYS-1 proteasomal degradation are affected by disruptions to

253 microtubule mediated trafficking. Though microtubule poisoning by Nocodazole and disruption of dynein function have opposite effects on SYS-1 centrosomal enrichment, either change in SYS-1 centrosomal enrichment may be indicative of a mis-localized regulatory process.

257 SYS-1 CEI, we investigated a combination of these treatments to observe the behavior of SYS-1 upon 258 further disruption to microtubule-mediated trafficking. Moreover, while both temperature upshift for $259 d h c-1(t s)$ and Nocodazole treatments significantly affect SYS-1 centrosomal localization, neither

260 treatment appears to completely disrupt microtubule mediated trafficking. Nocodazole-treated embryos 261 still exhibit some kinetochore microtubules (Fig. 3A) but can tolerate weakly poisoned microtubules for 
restrictive temperature that short-term temperature upshifts are required to establish spindles and maintain viability, suggesting some portion of centrosomal trafficking during mitosis still occurs. We therefore tested the combination of these treatments.

In contrast to embryos with GFP:SYS-1 driven by the strong early embryonic PIE-1 promoter (Fig.

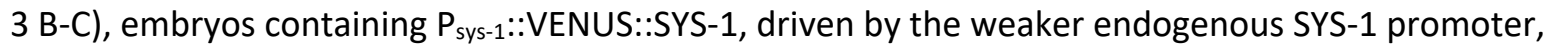
exhibit only a slight, non-significant, increase in CEI/TEI when treated with nocodazole (Fig. 3D, $1^{\text {st }}$ and $2^{\text {nd }}$ columns). However, when $\mathrm{P}_{\text {sys-1 }}:$ VENUS::SYS-1 centrosomal enrichment is challenged by the hypomorphic $d h c-1(t s)$ at the permissive temperature, nocodazole treatment is sufficient to increase SYS-1 centrosomal recruitment. The finding that sensitizing embryos for dynein trafficking defects is required to observe a nocodazole phenotype for $\mathrm{P}_{\text {sys- } 1:}: \mathrm{VENUS}:: \mathrm{SYS}-1$, but not for $\mathrm{P}_{\text {pie- } 1:: G F P:: S Y S-1}$ indicates that the difference in transgene expression uncover a differential nocodazole sensitivity in centrosomal recruitment of SYS-1. However, nocodazole-mediated increase in $\mathrm{P}_{\text {pie-1 } 1:: G F P:: S Y S-1}$ centrosomal enrichment is no longer observed when the nocodazole treated $d h c-1$ (ts) embryos are upshifted to the restrictive temperature, suggesting that the increased SYS-1 centrosomal enrichment seen in nocodazole treated embryos still requires dynein trafficking.

These observations describe a centrosomal relationship wherein treatments limiting the coverage of microtubule-mediated dynein trafficking (i.e. nocodazole treatment) increase centrosomal SYS-1 because of relatively inefficient or rare trafficking events for SYS-1 negative regulators, while transient severe disruptions to dynein trafficking processivity (dynein subunit RNAi depletion, dhc-1(ts) temperature upshift, upshift + nocodazole) reveal that SYS-1 centrosomal enrichment requires continual replenishment via a functional microtubule motor.

If the nocodazole-induced SYS-1 CEI increase described above is indeed because of a role for microtubule mediated trafficking in the centrosomal enrichment of a component of the SYS-1 
degradation pathway, we should expect proteolytic activity to be similarly mis-localized upon trafficking disruption. Therefore, we decided to test the idea that proteasomal trafficking to, and enrichment at, the centrosome was also perturbed in nocodazole-affected microtubule networks. We first attempted to localize the proteasome. While the centrosome has been demonstrated to exhibit proteolytic activity (Vora and Phillips 2015; Vora and Phillips 2016; Hames, Fry et al 2005; Peel, O'Connell et al 2012; mammalian proteasome subunits did not display any distinct localization pattern. An mCherry tag on proteasome subunit RPT-1 did demonstrate a kinetochore microtubule enrichment like that of both DHC-1 and SYS-1 but appeared absent from centrosomes. Inability to localize tagged RPT-1 may be due to a relatively low RPT-1 copy number per proteasome, or correspondingly low proteasome number, limiting its relative enrichment. Alternatively, fluorescently tagged proteins have shown evidence of degradation of the tag at a site of active proteolysis, particularly if such a tag limits proteasome activity (Huang, Lobel et al 2014). To circumvent this issue, we assayed for centrosomal proteasome activity indirectly by functionally testing an additional centrosomal target of the RPT-4 containing proteasome, the centriole duplicating kinase ZYG-1 (O'Connell, White et al 2001; Peel, O'Connell et al 2012). In addition, we assayed the centrosomal role of the C. elegans homolog of mammalian ECM29, ECPS-1, which is an adaptor protein that enhances local proteolytic activity of proteasomes via its function

303 linking the dynein motor complex and the proteasome and regulating 26S proteasome distribution 304 (Gorbea, Rechsteiner et al 2004; Gorbea, Rechsteiner et al 2010; Hsu, Cheng et al 2015; Ibañez-Vega, Yuseff et al 2021).

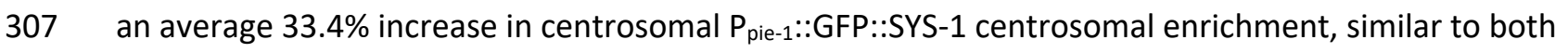


trafficking enhances the removal of SYS-1 from centrosomes as well. Given the role of ECM29 in depletion of microtubules (Nocodazole treatment). To determine if ECPS-1 knockdown is specific to SYS1 dysregulation, or perhaps plays a more general role in protein regulation, we assayed an additional target. The ZYG-1 protein, a regulatory kinase controlling centriolar duplication, is both centrosomally

317 enriched during mitosis and negatively regulated by the RPT-4 proteasome (Peel, O'Connell et al 2012).

318 Utilizing a fluorescently tagged ZYG-1 fragment demonstrated to mirror the localization and dynamics of the endogenous protein (Peters, O'Connell et al 2010), we demonstrated $20 \%$ and $27 \%$ increased accumulation of ZYG-1 in both proteasome knockdown rpt-4(RNAi) and ecps-1(RNAi) treatments, respectively. The similarity of phenotype between Nocodazole, ECPS-1 depletion, and RPT-4 proteasome knockdown implies a microtubule-mediated trafficking link between centrosomally localized proteins and their eventual degradation by the RPT-4 proteasome.

Since SYS-1 centrosomal recruitment depends on rapid degradation via the centrosomal proteasome (Vora and Phillips 2015), we assayed the turnover rate of centrosomal SYS-1 in ecps-1(RNAi) by FRAP. Assaying SYS-1 centrosomal turnover allowed us to assess the motility of centrosomal SYS-1.

327 Comparison of GFP::SYS-1 recovery after photobleach demonstrates that depletion of ECPS exhibits an 328 intermediate phenotype between proteasome depletion via rpt-4(RNAi) and negative controls (5G-G").

329 While the initial rate of ecps-1(RNAi) GFP::SYS-1 recovery was similar to that seen in wild type embryos, 330 the late recovery phase of ecps-1(RNAi) embryos showed an early SYS-1 recovery plateau, evidence of a 331 significantly reduced mobile fraction intermediate to wild type and that of proteasome depleted rpt$3324(R N A i)$ embryos (Fig. 4E,G). The recovery curve observed in ecps-1(RNAi) suggests that SYS-1 recovers 333 at an approximately wild type rate in ecps-1(RNAi) embryos but remains approximately as immobile as 
in proteasome-depleted embryos. Therefore, ECPS-1 depleted embryos appear to degrade SYS-1

335

incompletely, as observed in the RPT-4 proteasome depletion, but with sufficient protein turnover to enable wild type recruitment of newly trafficked SYS-1.

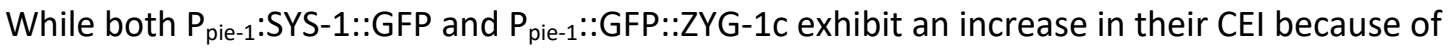
ECPS-1 depletion, their turnover rates at the centrosome are not similarly affected. The differential motility between SYS-1 and ZYG-1c may be due to diverging regulatory pathways en route to the RPT-4 proteasome, or differential sink volumes at the mitotic centrosome. Meanwhile, ECPS-1 depleted embryos may be unaffected in protein clearance per proteasome, but the delayed or partial localization of the centrosomal proteasome could result in hierarchical stabilization of a spatially or temporally inaccessible fraction consisting of highly expressed and continually trafficked proteins, like SYS-1.

\section{Dynein Trafficking is Required for Restriction of Wnt-Signaled Cell Fates}

Having established a two-fold role for SYS-1 regulation in microtubule-mediated trafficking, we investigated the role microtubule trafficking played in regulating SYS-1 dependent cell fate decisions. Since the SYS-1 centrosomal localization pattern has been seen across all examined SYS-1-expressing tissues (Phillips, Kimble et al 2007; Huang, Lin et al 2007; Baldwin and Phillips 2018; Lam and Phillips 2017), we investigated cell fate changes in embryonic and larval tissues across one embryonic and two larval SYS-1 dependent cell fate decisions. These included the transcriptional reporter of the W $\beta A$ target gene, end-1, in the establishment of SYS-1 dependent endoderm, and an anatomical marker for larval specification of the stem cell-like cells of the hypodermal seam and the germline stem cell niche. First, to directly assay the effect of centrosomal dysregulation of transcriptional activator SYS-1, we investigated the nuclear enrichment of GFP::SYS-1 after the Wnt-regulated EMS division (Bei, Mello et al 2002). In the EMS division, SYS-1 is enriched in the posterior E nucleus, but not that of the anterior MS (Fig. 5A-B). Analyzing nuclear enrichment of GFP-tagged SYS-1, we were able to observe increased nuclear GFP 
signal in both the E and MS daughters in analyzed trafficking mutants (Fig. 5C). Interestingly, daughters

affected by both ecps-1(RNAi) and dlc-1(RNAi) exhibit significant enhancement of nuclear enrichment in each of the two daughter cells (Fig 5C), suggesting that these treatments act negatively on SYS-1, despite opposing effect on SYS-1 centrosomal enrichment. Following loss of negative centrosomal regulation, these data suggest that daughter cell SYS-1 inheritance is increasing and thus affecting the bias of the EMS asymmetric division, increasing the likelihood of unsignaled daughter cells adopting the signaled endoderm cell fate.

Having established an increase in nuclear GFP::SYS-1 in dynein subunit depletion, even in cells not signaled to maintain activating SYS-1, we turned to the effect of SYS-1 expression on target gene activation. Specifically, we used the endogenous promoter of W $\beta A$ target gene end-1 to drive GFPtagged Histone 2B in order to evaluate the activity of SYS-1 immediate downstream targets as the signaled $E$ cell established the endoderm tissue expansion from 2 to 4 to 8 cells ( $E+1,2$, or 3 divisions, respectively). Normally, this strain exhibits additional expression of the end-1 promoter in $<10 \%$ of embryos, a frequency which decreases further when SYS-1 is depleted via RNAi (Fig. 5D). In embryos

371 depleted of either dynein subunits or RSA-2 by RNAi, however, we observed a different pattern. In these 372 embryos, misregulation via dynein depletion appeared to increasingly overwhelm SYS-1 negative 373 regulation in successive divisions, such that each generation after SYS-1 dependent specification 374 increasingly expresses the end-1 promoter driven endodermal reporter outside of the EMS lineage (Fig. 375 5D). For instance, in dhc-1(RNAi) we observe only $3 \%$ of embryos mis-expressing one cell division after 376 the specification of the $E$ cell type $(E+1)$, which increases to $25 \%$ by the next division ( $E+2)$, and over $83 \%$ 377 three divisions after specification $(E+3)$. 
their sensitivity to SYS-1 dependent gene activation (Baldwin, Phillips et al 2016; Baldwin and Phillips 2018; Lam and Phillips 2017; Mila, Putzke et al 2015). In wild-type animals, DTC lineages undergo asymmetric cell division during L1 to give rise to two DTCs, while sys-1 mutants and animals overexpressing SYS-1 show loss or gain of DTCs, respectively, due to symmetric cell division (Fig 6A) (Miskowski, Kimble et al 2001; Siegfried, Kimble et al 2004; Kidd, Kimble et al 2005; Chesney, Kimble et al 2009). To further sensitize these cells for changes in SYS-1 dependent cell fate changes, we examined strains containing additional, transgenic SYS-1 driven by the heat shock promoter. In animals that are not subjected to heat shock, dynein depletion only rarely induced development of more than the endogenous 2 distal tip or 16 seam cells (Fig. 6B, 7B). In heat shocked animals, excess SYS-1 protein can overwhelm canonical SYS-1 regulation to develop as many as 6 DTCs (Fig. 6D). While heat shock-induced overexpression is sufficient to cause a notable shift towards the signaled cell fate, dynein subunit depletion was able to significantly enhance the effect of heat shock (Fig. 6C). Depletion of DHC-1 or ECPS-1 at the time of the somatic gonadal precursor division was sufficient to induce rare but wild-typeunobserved additional DTCs ( $0.3 \%$ of animals for these 2 treatments, $\mathrm{N}=394,363$, respectively). Adding

394 heat shock-overexpressed SYS-1 enhanced the penetrance of ectopic DTCs, with DHC-1 and ECPS-1 depletion resulting in populations where $11.9 \%$ and $6.8 \%$ of animals, respectively, develop more than 2

DTCs. Depletion of RSA-2, uncoupling SYS-1 from centrosomes, and depletion of a dynein light chain,

397 DYLT-1, further enhanced these misspecifications, increasing the proportion of the population with ectopic DTCs from $26.6 \%$ in wild type to $33.6 \%$ or $51 \%$ after RNAi depletion, respectively (Fig. 6D).

400 (Fig 7A). Similarly, in the SC tissue, depletion of dynein light chains alone is sufficient to increase the 401 frequency of ectopic SCs from 3.3\% of larvae with ectopic SCs in control RNAi conditions to $11.7 \%$ or $40210 \%$ of animals as seen in DYLT-1 and DLC-1 depletions, respectively (Fig. 7B). These misspecifications are further exacerbated by SYS-1 overexpression, wherein more than $60 \%$ of the $d y / t-1$ (RNAi) larvae 
have greater than wild type seam cell numbers, developing as many as $21 \mathrm{SCs}$, (Fig. 7C). These

observations collectively support SYS-1 centrosomal enrichment by dynein as a mechanism to reinforce

SYS-1 function in specifying SYS-1-dependent cell fate decisions. Therefore, compromising the

in otherwise wildtype animals, but notably enhances the effects of ectopic SYS-1 accumulation.

Therefore, dynein-dependent centrosomal regulation of transcriptional coactivator SYS-1 increases the robustness of $W \beta A$-regulated cell fate decisions after $A C D$.

\section{Discussion}

Precise control of cell fate determinants is critical for normal development and homeostasis,

413 particularly in the rapidly dividing cells of the early embryo. Regulation of these factors includes the

414 distribution and inheritance of these determinants by daughter cells. Here we demonstrate that

415 centrosomal negative regulation of $C$. elegans $\beta$-catenin SYS-1 is enhanced during asymmetric division

416 by trafficking to centrosomes by the microtubule motor dynein. Depletion of individual subunits of the

417 dynein motor and associated proteins is sufficient to reduce the levels of centrosomal SYS-1, and

418 temporally specific ablation of dynein function by $d h c-1$ (ts) was sufficient to remove observable SYS-1

419 nearly completely and disproportionately from centrosomes. However, some methods of disrupting

420 dynein/microtubule trafficking result in an unexpected increase in centrosomal accumulation of SYS-1

421 protein. These increases, caused by microtubule disruption via Nocodazole and depletion of SYS-1

422 negative regulator adapter ECPS-1 phenocopy centrosomal enrichment seen in disruption of

423 proteasome activity, and maintain a SYS-1 immobile fraction like that seen in rpt-4(RNAi) depletion. We

424 therefore propose a model wherein both SYS-1 and regulatory components of the ubiquitin-proteasome

425 system, are dependent on microtubule-mediated trafficking to centrosomes by the dynein complex in

426 order to appropriately reinforce SYS-1 asymmetric regulation across daughter cells (Fig. 8). 
In this model, SYS-1 enriches at centrosomes and is degraded or marked for degradation before

428 leaving the centrosome. Rather than a static centrosomal enrichment, this model entails continual

429 trafficking by microtubule motors to maintain an observable centrosomal pool of SYS-1. The observable

430 CEI therefore measures a steady state of centrosomal enrichment at centrosomes, wherein SYS-1 is

431 localized to centrosomes more rapidly than it is marked and cleared. SYS-1 therefore becomes visibly

432 enriched at centrosomes compared to cytoplasmic levels. This model predicts that even subtle

433 disruptions to microtubule mediated trafficking could limit the ability of cells to maintain wild type SYS-1

434 centrosomal enrichment, assuming the SYS-1 clearance rate remains unchanged.

Interestingly, the rsa-2(RNAi) enhancement of kinetochore localization of SYS-1 in this model is consistent with 'traffic jams' observed in microtubule mediated trafficking (Leduc, Howard et al 2012). In

437 these cases, crowding motors by increasing the local concentration of motor proteins, introducing

438 microtubule-binding obstacles, or, importantly for our model, preventing motor dissociation limits the 439 activity of motor proteins (Nam and Epureanu, 2017; Ferro, Yildiz et al 2019). Evidence of traffic jams in

440 vivo have been shown in neuronal microtubule transport, as neuronal kinesin and its cargo

441 synaptotagmin accumulate when trafficking is disrupted by hypomorphic kinesin alleles. The disrupted

442 motor complexes then accumulate upstream of the trafficking impediment, in an accumulation of both

443 motor and cargo (Hurd and Saxton 1996).

444 The kinetochore localization of SYS-1 is consistent with a traffic jam model, thus making SYS-1 trafficking 445 by microtubule motors a more attractive model. Decreasing centrosomal SYS-1 localization by rsa-

4462 2(RNAi) increases SYS-1 protein in resulting daughter cells (Vora and Phillips 2015). A severely limited

447 centrosomal recruitment of SYS-1 in rsa-2(RNAi) may also suggest problematic SYS-1/motor protein

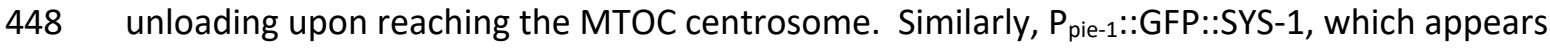

449 overexpressed compared to $P_{\text {sys-1 }}:$ VENUS::SYS-1, may exacerbate SYS- 1 traffic jams by increasing the 450 cytoplasmic SYS-1 pool. Indeed, the combination of $\mathrm{P}_{\text {pie-1 }}:: G F P:: S Y S-1$ and $r s a-2(R N A i)$ results in a further 
451 increase in the incidence of SYS-1 localization to kinetochore microtubules, suggesting increased SYS-1

452 levels coupled with decreased centrosomal capturing may reveal an otherwise transient pool of SYS-1 en

453 route to the MTOC centrosome. Inefficient SYS-1 capture in rsa-2(RNAi) embryos likely further crowds

454 SYS-1-containing microtubule motor complexes into 'traffic jams' because, in embryos lacking a

455 mechanism to anchor SYS-1 to the centrosome, SYS-1 is trafficked toward centrosomes more quickly

456 than it is captured there and processed. This shift results in an observable SYS-1 accumulation at regions

457 of high microtubule occupancy, such as kinetochore microtubules and the cell cortex, both of which are

458 increased after RSA-2 depletion.

Conversely, centrosomal protein processing by either post-translational modifications or degradation via the proteasome likely requires the trafficking of fewer, yet reusable enzymes. The 461 disparity between substrate vs enzyme trafficking requirements would suggest that clearance of 462 centrosomal substrates is less vulnerable to mild or short-term disruptions to trafficking than the maintenance of a centrosomal substrate pool. Upon long-term or severe disruptions to microtubule 464 trafficking, a trafficking requirement for clearance mechanisms becomes apparent. Our model predicts 465 that disruption of either SYS-1 localization (inefficient trafficking to the site of regulation) or disrupting 466 the localization of a member of the Ubiquitin-Proteasome System (UPS) (where SYS-1 is trafficked and 467 accumulates, but centrosomal regulation/degradation is ineffective) both result in increased SYS-1 468 dependent gene activation and differentiation in daughter cells, consistent with excessive inheritance.

469 While this system of centrosomal localization and clearance of SYS-1 does not appear necessary for most 470 W $\beta$ A differentiation events, disruptions to SYS-1 trafficking or processing limit the system's ability to 471 tolerate perturbations. That is, SYS-1 centrosomal localization generates robustness in SYS-1 dependent 472 cell fate decisions (Fig. -7) due to parallel trafficking mechanisms; disrupting either trafficking 473 mechanism results in excessive SYS-1 dependent activity in daughter cells of mothers with either 474 increased or decreased SYS-1 CEI. 
Why does no single dynein disruption treatment fully recapitulate the rsa-2(RNAi) phenotype?

476 While we see individuals within several dynein knockdown treatments with nearly rsa-2(RNAi)-like SYS-1

477 centrosomal uncoupling, these populations also include embryos with roughly wild type centrosomal

478 SYS-1 enrichment. However, given the severely deleterious nature of dynein subunit loss-of-functions

479 (Sönnichsen, Neumann et al 2005) and the variability of feeding RNAi knockdowns as compared to null

480 mutant strains (Tavernarakis, Driscoll et al 2000), it seems likely that our significant decreases in SYS-1

481 centrosomal recruitment represent a phenotypically incomplete knockdown. Surviving embryos then

482 only exhibit hypomorphic dynein function, insufficient to induce catastrophic defects in spindle

483 assembly but sufficient to affect SYS-1 centrosomal enrichment. However, it is also possible that there is

484 partial redundancy between the SYS-1 relevant functions of the dynein motor. Introducing dhc-1(ts)

485 overcomes either incomplete knockdown or redundancy since a thorough dynein disruption is able to

486 robustly uncouple centrosomal SYS-1.

Why, then, do we observe an increase in centrosomal SYS-1 steady state upon either long term

488 lower dose nocodazole-mediated knockdowns of trafficking or by RNAi depletion of trafficking subunit

489 ECPS-1? In response to these data (Fig. 3), we propose the additional trafficking of a SYS-1 negative

490 regulatory component to this model. Disrupting the trafficking of centrosomal SYS-1 negative regulators

491 should result in a previously unseen increase in centrosomal SYS-1 steady state while maintaining a

492 similar effect on downstream gene expression and cell fate decisions, as seen above (Fig. 3-7). However,

493 the difference in SYS-1 enrichment between most dynein depletions and that of ECPS-1 or nocodazole

494 treatment suggests a differential efficiency in trafficking or centrosomal capture rate between SYS-1 and

495 its negative regulators, such as the proteasome. Most disruptions to the dynein motor that reduce SYS-1

496 centrosomal accumulation, mediated by maternally fed RNAi, are consistent but likely incomplete

497 because of their requirement for oogenesis and early embryonic viability. Relatively mild dynein

498 knockdowns resulting in viable embryos, therefore, are likely able to form dynein complexes, but at a far 
reduced rate. These dynein-limited embryos should therefore decrease the rate of continual SYS-1

500 trafficking while not preventing the centrosomal localization of an occasional regulatory components,

501 perhaps of the Ubiquitin-Proteasome System (UPS). The reusable tagging or proteolytic machinery is

502 likely sufficient to enzymatically process SYS-1 that still localizes to the centrosome, but the rate of

503 incoming SYS-1 is significantly reduced. Similarly, depletion of dynein function by dhc-1(ts) effectively

504 depletes all dynein function but can only do so for brief portions of mitosis without inducing mitotic

505 spindle collapse.

While dynein depletions limit the volume of trafficking by limiting the number of properly

constructed dynein motors, they do not limit the ability of an individual, functional dynein motor to

traffic. The experimental conditions that increase the steady state of centrosomal SYS-1, Nocodazole

and ecps-1(RNAi), instead limit the scope of dynein cargo trafficking. ECPS-1, putative adapter between

dynein and the proteasome, increases the steady state of both SYS-1 and additional centrosomal

proteasome target ZYG-1. Increased enrichment of both proteins is consistent with the conserved role

514 processivity of dynein complexes that form on the comparatively limited microtubule network.

515 However, it does severely affect the reach of the microtubule network itself (Fig. 3A). Our data therefore

516 suggests that the proteasome requires less continual reinforcement by microtubule-mediated trafficking

517 but does require more extensive microtubule formation than is allowed in our Nocodazole-treated

518 embryos.

A role for trafficking in both SYS- 1 and its regulatory components, then, suggests a differentially 
524 centrosomal enrichment to be overcome by its centrosomal processing, reducing the SYS-1 CEI by

525 slowing the transfer of SYS-1 from cytoplasm to centrosome for degradation. By comparison, the

526 proteasome or ubiquitin ligases endure at the centrosome, continually processing centrosomal targets.

527 Our proposed model therefore predicts that short term or partial disruptions to the dynein motor (Fig.

528 8, red inset) allow for the eventual establishment of a centrosomal proteolysis pathway, which may

529 affect both SYS-1 and proteasome localization, but nevertheless result in decreasing steady state levels

530 of centrosomal SYS-1 (and presumably other proteasome targets that require active trafficking) as

531 occasional proteasome centrosomal localization overcomes the impaired localization of SYS-1.

532 Conversely, long term systemic disruptions to microtubule-mediated motors like that seen in

533 Nocodazole treatment may still capture SYS-1 in their comparatively limited microtubule network, while

534 being disproportionately deprived of the larger proteasome complex (Fig. 8, blue inset).

Our model that both SYS-1 and its regulators are trafficked was supported but the identification

536 of a trafficking component that affects one but not both arms of our trafficking model. Most of our

537 perturbations, generally affecting components of the dynein machinery, may exert their effects by

538 either detaching SYS-1 from the motor or by sabotaging all function of the motor complex. However, the

539 specificity of our two transport mechanisms was exhibited by one dynein-protein interaction, via

540 depletion of ECPS-1/the putative ECM29 subunit of the motor. Unlike the other subunits presenting

541 strong SYS-1 phenotypes, ECM29 has not yet been implicated in dynein function beyond its adaptor role

542 but has notable effects on proteasome localization and response to stress (Gorbea, Rechsteiner et al

543 2010; Haratake, Chiba et al 2016). Because ecps-1(RNAi) phenocopies the centrosomal GFP::SYS-1

544 accumulation seen in proteasome knockdown via rpt-4(RNAi) as well as microtubule network disruption

545 via Nocodazole treatment, we propose that these observations support a role for proteasomal

546 localization as well as function for proper centrosomal clearance of SYS-1 (Fig. 8). 
While this model and our experiments to test it have been largely centered on SYS- $1 / \beta$-catenin,

548

549

550

551

552

553

554

555

556

557

558

559

560

561

562

563

564

565

566

567

568

569

many developmental and temporally regulated proteins are known to pass through the centrosome

(Vora and Phillips 2016; Brown, Welch et al 1994; Johnston, Kopito et al 1998; Huang and Raff 1999;

Reck-Peterson, Carter et al 2018), as the centrosomal proteasome is far from SYS-1-specific. It is not

unreasonable, then, to presume that other proteins may benefit from dynein trafficking to something as

short-lived and yet influential to daughter cell inheritance as the mitotic centrosome. Particularly early

in eukaryotic development, subtle changes in the inheritance of cell fate determinants like SYS-1 can

have a profound effect on the ability of an organism to respond to environmental insults. For example, the asymmetric nuclear localization of SYS-1 indicative of differential cell fate has been shown to arise early, less than 70 seconds after the establishing division (Baldwin and Phillips 2014; Vora and Phillips 2015). We therefore propose that an active dynein mediated trafficking, at least for cell fate determinants like SYS-1, could provide a universal buffering against excessive inheritance and consequentially rare but deleterious cell fate changes.

\section{Materials and Methods}

\section{Strains}

Strains were maintained on Op50-inoculated NGM plates at $15^{\circ}$ (BTP51, BTP220) or $25^{\circ} \mathrm{C}$

(TX964, BTP51, LP563, TX691) using typical C. elegans methods. These strains contained the following transgenes and alleles: N2 (wild type); TX964 (unc-119(ed3) III; him-3(e1147) IV; teis98 [Ppie-

1::GFP::SYS-1]); BTP51 (unc-119(ed3) III; tjls71 [pie-1 promoter::mCherry::H2B, pie-1 promoter::2x

mCherry::tbg-1, unc-119(+)]; qls95 III) ; BTP220 (unc-119(ed3) III; tjls71 [pie-1 promoter::mCherry::H2B, pie-1 promoter::2x mCherry::tbg-1, unc-119(+)]; qls95 III; dhc-1(or195) I.) ; TX691 (unc-119(ed3) III; tels46 [pRL1417; end-1p::GFP::H2B + unc-119(+)]) ; OC341 (unc-119(ed3) III; bsls8[pMS5.1:unc -119(+) pie -1- gfp-zyg-1C-terminus]); BTP93 (wls78[SCMp::GFP + ajm-1p::GFP + F58E10 (cosmid) + unc-119(+)] 
IV; uiwEx22[pHS::sys-1]); BTP97 (qls56[lag-2p::GFP + unc-119(+)] V;uiwls3[pHS::sys-1]); AZ235 (unc-

119(ed3) III; ruls48[Ppie-1::gfp::tbb-1]; TH107 (P pie-1::RSA-2:: GFP)

574 plasmid with a T7-flanked target gene insert. Most of these were obtained from a library supplied by

575 Ahringer (Kamath and Ahringer 2003). Those which were otherwise obtained (dlc-1) used target gene-

576 flanking primers with C. elegans cDNA to generate a new insert. These bacteria were used to seed IPTG-

577 containing, inducing plates as described previously. Worms were generally plated to RNAi after Sodium

578 Hypochlorite Synchronization as L1's. More deleterious knockdowns (dlc-1, dhc-1) were washed from

579 OP50 plates at 24 hours before imaging (L3/L4) or 12 hours before imaging (late L4/early adult)

580 (Timmons 2006; Bekas and Phillips 2020). DLC-1 was derived using RT primers and digested with Xho I and HIND III SEQUENCING 7/31

\section{Compound Microscopy, CEI, and Image Processing}

To obtain samples, mothers were anesthetized with $250 \mu \mathrm{M}$ levamisole and dissected via a clean surgical blade. Embryos were then transferred to a 2\% agarose pad and imaged on a Zeiss Axio mitotic P1 cell of the early embryo during anaphase, at approximately similar inter-polar distance (Fig. 


\section{Temperature Sensitive Allele Imaging}

BTP51 and BTP220 were maintained together at $15^{\circ} \mathrm{C}$ and were imaged as above. Ambient

temperature in the microscope room was decreased to $\sim 18^{\circ} \mathrm{C}$ for 'permissive' imaging and upshifted to

596 'restrictive' $26^{\circ} \mathrm{C}$ by moving slides to a Pecon Tempcontroller 2000-2 heated stage. Embryos were

597 imaged at the two temperatures sequentially, with approx. 45 seconds between upshift and image, or

598 were imaged as populations at either the permissive or restrictive temperature.

599

600

601

602

603

604

605

606

607

608

609

610

611

612

613

614

\section{Nocodazole Treatment}

In order to treat embryos before formation of the drug-impermeable eggshell, we exposed late L4's to a low dose (approx. $83 \mu \mathrm{M}, 25 \mu \mathrm{g} / \mathrm{mL}$ ) nocodazole dissolved in 2\% DMSO for 16 hours. Embryos were then concentrated by one minute centrifugation at $1000 \times \mathrm{x}$ and imaged as described above.

Permeabilization of embryos was performed as described in Carvalho, Oegema et al 2011. Imaging was done as described above, but included $4 \%$ sucrose and $0.1 \mathrm{M} \mathrm{NaCl}$ to the $2 \%$ agar pads to bolster embryo stability (Greenan, Hyman et al 2010; Gönczy, Schnabel et al 1999).

\section{Confocal Microscopy and Fluorescence Recovery After Photobleach}

Confocal microscopy was performed via a Leica SP8 HyD detector system. The objective used was a $63 x$ HC PL APO CS2 objective with N.A. = 1.4, utilizing type F immersion oil. Each image analyzed consisted of the sum of $35 \mathrm{z}$-images across the embryo, each slice approximately $0.35 \mu \mathrm{M}$ thick. FRAP was performed on the same system. Each embryo was imaged twice before bleaching, beginning as soon as possible after NEBD. The ROI, an approximately $5 \mu \mathrm{m}$ square, was then bleached at an intermediate focal plane with $100 \%$ laser power for 100 iterations. Images were taken as above, once every 7 seconds. Fluorescence intensity was measured on summed slice Z-projections in FIJI via the mean fluorescence intensity of ROIs drawn around relevant locales - either centrosome or the entire 
615 dividing cell. Recovery was assayed by subtracting the ROI post-bleach intensity from each

616 measurement and evaluating it as a percentage of the pre-bleached centrosomal intensity.

\section{Heat Shock-Induced SYS-1 Overexpression}

619 timepoints to drive SYS-1 overexpression concurrently with signaled cell fate specification. For DTCS,

620 BTP97 was subjected to 1-hour heat shock, 30-minutes recovery, and 30-minutes additional heat shock

621 beginning 11.5 hours after plating. For SCs, BTP93 was subjected to 2-hour heat shock, 30-minute

622 recovery, and additional 1-hour heat shock beginning 26 hours after plating. Both tissue types were

623 assayed 50 hours after plating.

\section{Acknowledgements}

We thank Kimberly Bekas and Amy Clemons for helpful comments on the manuscript. Strains

626 were provided by the Caenorhabditis Genetics Center, which is funded by the National Institutes of

627 Health (NIH) Office of Research Infrastructure Programs [P40 OD01440], or gifts from the laboratory of

628 Tony Hyman (TH107) and Dr. Kevin F. O'Connell at the NIDDK (OC341). This work was supported by NIH 629 award NIH GM114007 (B.T.P.). 


\section{References}

637

638

639

640

641

642

643

644

645

646

647

648

649

650

651

652

653

654

655

656

657

658

Amsterdam, A., F. Pitzer and W. Baumeister (1993). "Changes in intracellular localization of proteasomes

in immortalized ovarian granulosa cells during mitosis associated with a role in cell cycle control." Proc

Natl Acad Sci U SA 90(1): 99-103.

Baldin, V., M. Militello, Y. Thomas, C. Doucet, W. Fic, S. Boireau, I. Jariel-Encontre, M. Piechaczyk, E.

Bertrand, J. Tazi and O. Coux (2008). "A novel role for PA28gamma-proteasome in nuclear speckle

organization and SR protein trafficking." Mol Biol Cell 19(4): 1706-1716.

Baldwin, A. T., A. M. Clemons and B. T. Phillips (2016). "Unique and redundant beta-catenin regulatory

roles of two Dishevelled paralogs during C. elegans asymmetric cell division." J Cell Sci 129(5): 983-993.

Baldwin, A. T. and B. T. Phillips (2014). "The tumor suppressor APC differentially regulates multiple beta-

catenins through the function of axin and CKlalpha during C. elegans asymmetric stem cell divisions." $\underline{\mathrm{J}}$

Cell Sci 127(Pt 12): 2771-2781.

Baldwin, A. T. and B. T. Phillips (2018). Chapter 3 - Cell Polarity and Asymmetric Cell Division by the Wnt Morphogen. Cell Polarity in Development and Disease. P. Michael Conn. Boston, Academic Press: 61-

102.

Bei, Y., J. Hogan, L. A. Berkowitz, M. Soto, C. E. Rocheleau, K. M. Pang, J. Collins and C. C. Mello (2002).

"SRC-1 and Wnt signaling act together to specify endoderm and to control cleavage orientation in early

C. elegans embryos." Dev Cell 3(1): 113-125.

Bekas, K. N. and B. T. Phillips (2020). "Generating reliable hypomorphic phenocopies by RNAi using long dsRNA as diluent." MicroPubl Biol 2020.

Ben-Yaakov, K., S. Y. Dagan, Y. Segal-Ruder, O. Shalem, D. Vuppalanchi, D. E. Willis, D. Yudin, I. Rishal, F. Rother, M. Bader, A. Blesch, Y. Pilpel, J. L. Twiss and M. Fainzilber (2012). "Axonal transcription factors signal retrogradely in lesioned peripheral nerve." EMBO J 31(6): 1350-1363. 
Bullock, S. L. and D. Ish-Horowicz (2001). "Conserved signals and machinery for RNA transport in

Drosophila oogenesis and embryogenesis." Nature 414(6864): 611-616.

661

Carminati, J. L. and T. Stearns (1997). "Microtubules orient the mitotic spindle in yeast through dynein-

Chesney, M. A., N. Lam, D. E. Morgan, B. T. Phillips and J. Kimble (2009). "C. elegans HLH-

2/E/Daughterless controls key regulatory cells during gonadogenesis." Dev Biol 331(1): 14-25.

671 Fabunmi, R., W. Wigley, P. Thomas and G. DeMartino (2000). "Activity and Regulation of the

672 Centrosome-associated Proteasome." Journal of Biological Chemistry 275: 409-413.

673 Fan, J., Q. Zhang, H. Tochio, M. Li and M. Zhang (2001). "Structural basis of diverse sequence-dependent 674 target recognition by the 8 kDa dynein light chain." J Mol Biol 306(1): 97-108.

675 Ferenz, N. P., A. Gable and P. Wadsworth (2010). "Mitotic functions of kinesin-5." Seminars in cell \& 676 developmental biology 21(3): 255-259.

677 Fuchs, E. and T. Chen (2013). "A matter of life and death: self-renewal in stem cells." EMBO Rep 14(1): $678 \quad 39-48$.

679 Gao, F. J., L. Shi, T. Hines, S. Hebbar, K. L. Neufeld and D. S. Smith (2017). "Insulin signaling regulates a 680 functional interaction between adenomatous polyposis coli and cytoplasmic dynein." Mol Biol Cell 28(5): 587-599. 
682

683

684

685

686

687

688

689

690

691

692

693

694

695

696

697

698

699

700

701

702

703

Geyer, F. C., M. Lacroix-Triki, K. Savage, M. Arnedos, M. B. Lambros, A. MacKay, R. Natrajan and J. S. Reis-Filho (2011). "beta-Catenin pathway activation in breast cancer is associated with triple-negative phenotype but not with CTNNB1 mutation." Mod Pathol 24(2): 209-231.

Gómez-López, S., R. G. Lerner and C. Petritsch (2014). "Asymmetric cell division of stem and progenitor cells during homeostasis and cancer." Cell Mol Life Sci 71(4): 575-597.

Gönczy, P., H. Schnabel, T. Kaletta, A. D. Amores, T. Hyman and R. Schnabel (1999). "Dissection of Cell

Division Processes in the One Cell Stage Caenorhabditis elegans Embryo by Mutational Analysis." Journal of Cell Biology 144(5): 927-946.

Gorbea, C., G. M. Goellner, K. Teter, R. K. Holmes and M. Rechsteiner (2004). "Characterization of mammalian Ecm29, a $26 \mathrm{~S}$ proteasome-associated protein that localizes to the nucleus and membrane vesicles." J Biol Chem 279(52): 54849-54861.

Gorbea, C., G. Pratt, V. Ustrell, R. Bell, S. Sahasrabudhe, R. E. Hughes and M. Rechsteiner (2010). "A protein interaction network for Ecm29 links the $26 \mathrm{~S}$ proteasome to molecular motors and endosomal components." J Biol Chem 285(41): 31616-31633.

Greenan, G., C. P. Brangwynne, S. Jaensch, J. Gharakhani, F. Jülicher et al., 2010 Centrosome size sets mitotic spindle length in Caenorhabditis elegans embryos. Curr Biol 20: 353-358.

Hames, R. S., R. E. Crookes, K. R. Straatman, A. Merdes, M. J. Hayes, A. J. Faragher and A. M. Fry (2005).

"Dynamic recruitment of Nek2 kinase to the centrosome involves microtubules, PCM-1, and localized proteasomal degradation." Mol Biol Cell 16(4): 1711-1724.

Hamill, D. R., A. F. Severson, J. C. Carter and B. Bowerman (2002). "Centrosome maturation and mitotic spindle assembly in C. elegans require SPD-5, a protein with multiple coiled-coil domains." Dev Cell 3(5): 673-684. 
704

705

706

707

708

709

710

711

712

713

714

715

716

717

718

719

720

721

722

723

724

725

Hanz, S., E. Perlson, D. Willis, J. Q. Zheng, R. Massarwa, J. J. Huerta, M. Koltzenburg, M. Kohler, J. vanMinnen, J. L. Twiss and M. Fainzilber (2003). "Axoplasmic importins enable retrograde injury signaling in lesioned nerve." Neuron 40(6): 1095-1104.

Harada, A., Y. Takei, Y. Kanai, Y. Tanaka, S. Nonaka and N. Hirokawa (1998). "Golgi vesiculation and lysosome dispersion in cells lacking cytoplasmic dynein." J Cell Biol 141(1): 51-59.

Haratake, K., A. Sato, F. Tsuruta and T. Chiba (2016). "KIAA0368-deficiency affects disassembly of 26S proteasome under oxidative stress condition." J Biochem 159(6): 609-618.

He, T. C., A. B. Sparks, C. Rago, H. Hermeking, L. Zawel, L. T. da Costa, P. J. Morin, B. Vogelstein and K. W. Kinzler (1998). "Identification of c-MYC as a target of the APC pathway." Science 281(5382): 1509-1512.

Horgan, C. P., S. R. Hanscom, R. S. Jolly, C. E. Futter and M. W. McCaffrey (2010). "Rab11-FIP3 links the Rab11 GTPase and cytoplasmic dynein to mediate transport to the endosomal-recycling compartment." J Cell Sci 123(Pt 2): 181-191.

Hsu, M. T., C. L. Guo, A. Y. Liou, T. Y. Chang, M. C. Ng, B. I. Florea, H. S. Overkleeft, Y. L. Wu, J. C. Liao and P. L. Cheng (2015). "Stage-Dependent Axon Transport of Proteasomes Contributes to Axon

Development." Dev Cell 35(4): 418-431.

Huang, L., D. Pike, D. E. Sleat, V. Nanda and P. Lobel (2014). "Potential pitfalls and solutions for use of fluorescent fusion proteins to study the lysosome." PLoS One 9(2): e88893.

Huang, S., P. Shetty, S. M. Robertson and R. Lin (2007). "Binary cell fate specification during C. elegans embryogenesis driven by reiterated reciprocal asymmetry of TCF POP-1 and its coactivator beta-catenin SYS-1." Development 134(14): 2685-2695.

Hutagalung, A. H. and P. J. Novick (2011). "Role of Rab GTPases in membrane traffic and cell physiology." Physiol Rev 91(1): 119-149. 
Ibañez-Vega, J., F. Del Valle, J. J. Sáez, F. Guzman, J. Diaz, A. Soza and M. I. Yuseff (2021). "Ecm29-

Jha, R. and T. Surrey (2015). "Regulation of processive motion and microtubule localization of

Kidd, A. R., 3rd, J. A. Miskowski, K. R. Siegfried, H. Sawa and J. Kimble (2005). "A beta-catenin identified

743 Leduc, C., K. Padberg-Gehle, V. Varga, D. Helbing, S. Diez and J. Howard (2012). "Molecular crowding creates traffic jams of kinesin motors on microtubules." Proceedings of the National Academy of

746 Leggett, D. S., J. Hanna, A. Borodovsky, B. Crosas, M. Schmidt, R. T. Baker, T. Walz, H. Ploegh and D. 747 Finley (2002). "Multiple associated proteins regulate proteasome structure and function." $\underline{\text { Mol Cell }}$ 

and stabilizes beta-catenin at mitotic centrosomes downstream of Plk1." Mol Biol Cell 25(7): 977-991. Mikenberg, I., D. Widera, A. Kaus, B. Kaltschmidt and C. Kaltschmidt (2007). "Transcription factor NFkappaB is transported to the nucleus via cytoplasmic dynein/dynactin motor complex in hippocampal neurons." PLoS One 2(7): e589.

"Asymmetric Wnt Pathway Signaling Facilitates Stem Cell-Like Divisions via the Nonreceptor Tyrosine Kinase FRK-1 in Caenorhabditis elegans." Genetics 201(3): 1047-1060.

Mizumoto, K. and H. Sawa (2007). "Cortical beta-catenin and APC regulate asymmetric nuclear beta- 
772

773

774

775

776

777

778

779

780

781

782

783

784

785

786

787

788

789

790

791

792

793

794
O'Rourke, S. M., M. D. Dorfman, J. C. Carter and B. Bowerman (2007). "Dynein modifiers in C. elegans: light chains suppress conditional heavy chain mutants." PLoS Genet 3(8): e128.

Peel, N., M. Dougherty, J. Goeres, Y. Liu and K. F. O'Connell (2012). "The C. elegans F-box proteins LIN-23 and SEL-10 antagonize centrosome duplication by regulating ZYG-1 levels." J Cell Sci 125(Pt 15): 35353544.

Peters, N., D. E. Perez, M. H. Song, Y. Liu, T. Muller-Reichert, C. Caron, K. J. Kemphues and K. F. O'Connell (2010). "Control of mitotic and meiotic centriole duplication by the Plk4-related kinase ZYG-1." J Cell Sci 123(Pt 5): 795-805.

Phillips, B. T., A. R. Kidd, 3rd, R. King, J. Hardin and J. Kimble (2007). "Reciprocal asymmetry of SYS1/beta-catenin and POP-1/TCF controls asymmetric divisions in Caenorhabditis elegans." Proc Natl Acad Sci U SA 104(9): 3231-3236.

Pintard, L. and B. Bowerman (2019). "Mitotic Cell Division in Caenorhabditis elegans." Genetics 211(1): 35-73.

Pinto, D., A. Gregorieff, H. Begthel and H. Clevers (2003). "Canonical Wnt signals are essential for homeostasis of the intestinal epithelium." Genes Dev 17(14): 1709-1713.

Priyanga, J., G. Guha and D. Bhakta-Guha (2021). "Microtubule motors in centrosome homeostasis: A target for cancer therapy?" Biochim Biophys Acta Rev Cancer 1875(2): 188524.

Reck-Peterson, S. L., W. B. Redwine, R. D. Vale and A. P. Carter (2018). "The cytoplasmic dynein transport machinery and its many cargoes." Nat Rev Mol Cell Biol 19(6): 382-398.

Reilein, A., D. Melamed, K. S. Park, A. Berg, E. Cimetta, N. Tandon, G. Vunjak-Novakovic, S. Finkelstein and D. Kalderon (2017). "Alternative direct stem cell derivatives defined by stem cell location and graded Wnt signalling." Nat Cell Biol 19(5): 433-444.

Reya, T. and H. Clevers (2005). "Wnt signalling in stem cells and cancer." Nature 434(7035): 843-850. 
Sawa, H. (2012). "Control of cell polarity and asymmetric division in C. elegans." Curr Top Dev Biol 101:

55-76.

797 Schlaitz, A. L., M. Srayko, A. Dammermann, S. Quintin, N. Wielsch, I. MacLeod, Q. de Robillard, A. Zinke,

J. R. Yates, 3rd, T. Müller-Reichert, A. Shevchenko, K. Oegema and A. A. Hyman (2007). "The C. elegans

RSA complex localizes protein phosphatase $2 \mathrm{~A}$ to centrosomes and regulates mitotic spindle assembly."

800

Cell 128(1): 115-127.

801

Schmidt, R., L. E. Fielmich, I. Grigoriev, E. A. Katrukha, A. Akhmanova and S. van den Heuvel (2017). "Two

populations of cytoplasmic dynein contribute to spindle positioning in C. elegans embryos." $\underline{\mathrm{J} \text { Cell Biol }}$

803

216(9): 2777-2793.

804

Shtutman, M., J. Zhurinsky, I. Simcha, C. Albanese, M. D'Amico, R. Pestell and A. Ben-Ze'ev (1999). "The

805

cyclin D1 gene is a target of the beta-catenin/LEF-1 pathway." Proc Natl Acad Sci U S A 96(10): 5522-

806

5527.

807

Siegfried, K. R., A. R. Kidd, M. A. Chesney and J. Kimble (2004). "The <em>sys-1</em> and <em>sys-

<em>Caenorhabditis elegans</em> Gonad." Genetics 166(1): 171-186.

812 Stamford, M. Winzi, R. Heinkel, M. Roder, J. Finell, H. Hantsch, S. J. Jones, M. Jones, F. Piano, K. C.

813 Gunsalus, K. Oegema, P. Gonczy, A. Coulson, A. A. Hyman and C. J. Echeverri (2005). "Full-genome RNAi

814 profiling of early embryogenesis in Caenorhabditis elegans." Nature 434(7032): 462-469.

815 Sugioka, K., K. Mizumoto and H. Sawa (2011). "Wnt regulates spindle asymmetry to generate

816 asymmetric nuclear beta-catenin in C. elegans." Cell 146(6): 942-954.

817 Sunchu, B. and C. Cabernard (2020). "Principles and mechanisms of asymmetric cell division."

818 Development 147(13). 

microtubules control redistribution of dynein at the cell cortex to facilitate spindle positioning." Cell Cycle 13(7): 1162-1170.

822 Tavernarakis, N., S. L. Wang, M. Dorovkov, A. Ryazanov and M. Driscoll (2000). "Heritable and inducible genetic interference by double-stranded RNA encoded by transgenes." Nat Genet 24(2): 180-183. Tejpar, S., F. Nollet, C. Li, J. S. Wunder, G. Michils, P. dal Cin, E. Van Cutsem, B. Bapat, F. van Roy, J. J. dysregulation in sporadic aggressive fibromatosis (desmoid tumor)." Oncogene 18(47): 6615-6620. Tetsu, O. and F. McCormick (1999). "Beta-catenin regulates expression of cyclin D1 in colon carcinoma cells." Nature 398(6726): 422-426. Timmons, L. (2006). "Delivery methods for RNA interference in C. elegans." Methods Mol Biol 351: 119125. and A. Couve (2014). "Transport along the dendritic endoplasmic reticulum mediates the trafficking of GABAB receptors." J Cell Sci 127(Pt 15): 3382-3395. Vora, S. and B. T. Phillips (2015). "Centrosome-Associated Degradation Limits beta-Catenin Inheritance by Daughter Cells after Asymmetric Division." Curr Biol 25(8): 1005-1016.

836 Vora, S. M., J. S. Fassler and B. T. Phillips (2020). "Centrosomes are required for proper beta-catenin 837 processing and Wnt response." Mol Biol Cell 31(17): 1951-1961.

838 Vora, S. M. and B. T. Phillips (2016). "The benefits of local depletion: The centrosome as a scaffold for 839 ubiquitin-proteasome-mediated degradation." Cell Cycle 15(16): 2124-2134.

840 Wigley, W. C., R. P. Fabunmi, M. G. Lee, C. R. Marino, S. Muallem, G. N. DeMartino and P. J. Thomas 841 (1999). "Dynamic association of proteasomal machinery with the centrosome." J Cell Biol 145(3): 481490. 
843 Wójcik, C. and G. N. DeMartino (2003). "Intracellular localization of proteasomes." The International

$844 \quad$ Journal of Biochemistry \& Cell Biology 35(5): 579-589.

845 Wozniak, M. J., B. Bola, K. Brownhill, Y. C. Yang, V. Levakova and V. J. Allan (2009). "Role of kinesin-1 and

846 cytoplasmic dynein in endoplasmic reticulum movement in VERO cells." J Cell Sci 122(Pt 12): 1979-1989.

847 Wühr, M., E. S. Tan, S. K. Parker, H. W. Detrich, 3rd and T. J. Mitchison (2010). "A model for cleavage

848 plane determination in early amphibian and fish embryos." Curr Biol 20(22): 2040-2045.

849 Yang, L., X. Wu, Y. Wang, K. Zhang, J. Wu, Y. C. Yuan, X. Deng, L. Chen, C. C. Kim, S. Lau, G. Somlo and Y.

850 Yen (2011). "FZD7 has a critical role in cell proliferation in triple negative breast cancer." Oncogene

$85130(43):$ 4437-4446.

852 Zhunussova, G., G. Afonin, S. Abdikerim, A. Jumanov, A. Perfilyeva, D. Kaidarova and L. Djansugurova

853 (2019). "Mutation Spectrum of Cancer-Associated Genes in Patients With Early Onset of Colorectal

854 Cancer." Front Oncol 9: 673.

855

856

857

858

859

860

861 


\section{Figure 1:}

865

866

867

868

869

870

871

872

873

874

875

876 877 increase centrosomal GFP::SYS-1 notably above the bulk of cytoplasmic representation. C) Example 878 VENUS::SYS-1 CEI (C) and CEI/TEI ( $\left.C^{\prime}\right)$ of controls and indicated dynein light chain depletions. Width of

879

880

881

882

Rationale and methodology for microtubule motor RNAi Screen. A) GFP::SYS-1 can be occasionally observed at regions corresponding to DHC-1 mitotic accumulation. This is particularly enhanced in centrosomally uncoupled rsa-2(RNAi) embryos. The frequency of GFP::SYS-1 enrichment at these locales in lacZ(RNAi) and rsa-2(RNAi) treatment is distinguished by color. $\left.\mathrm{A}^{\prime}\right)$ GFP::SYS-1 at kinetochore microtubule (white arrowhead) and cortex(black arrowhead) of the dividing C. elegans early embryo. B) Demonstration of CEI and TEI calculation. CEI was calculated in FIJI by subtracting the mean pixel intensity of a cytoplasmic ROI encompassing the proximal half of the dividing cell, approximated by the light blue square, from a circular centrosome ROI, approximated by the white square. B') Expanded view of ROI's as defined in B. B") Graphical representation of the raw centrosomal and CEl adjusted fluorescence intensity for the control (Left) and Affected (right) embryos in B. Control (lacZ(RNAi) treated) exhibits both a high raw centrosomal GFP::SYS-1 and CEI. Affected (rsa-2(RNAi) treated) exhibits an intermediate raw GFP::SYS-1, but severely decreased CEI, better reflecting its visual failure to the violin plot corresponds to a histogram of observation density. The median is indicated by the central white dot, with the interquartile range indicated by the thick black line, and 1.5x additional Interquartile range indicated by the thin black line. $\mathrm{N}=\operatorname{lacZ}(R N A i), 72$; dlc-1(RNAi), 74; dylt-1(RNAi), 52; rsa-2(RNAi), $22{ }^{*}-p<0.05,{ }^{* *}-p<0.01,{ }^{* * *}-p<0.001$ by Chi-square test (A) or Student's t-test (C- $\left.C^{\prime}\right)$.

\section{Figure 2:}



upshift. D) SYS-1 CEI and D') SYS-1 CEI per TEI in embryos containing dhc-1(ts), to control for differences $\mathrm{p}<0.05, * *-p<0.01, * * *-p<0.001$ by Student's t-test.

Figure 3: indicate astral microtubules (left) and the corresponding lack or dysregulated region in nocodazole treatment (right) B) Quantification of, and C) Representative images of similarly treated GFP::SYS-1

897 embryos. For $\mathrm{B}, \mathrm{N}=36$ DMSO and 46 nocodazole treated embryos. ${ }^{*}-\mathrm{p}<0.05,{ }^{* *}-\mathrm{p}<0.01, * * *-p<0.001$

898 by Student's t-test. D) CEI/TEI as evaluated with combinatorial Wildtype (WT) or dhc-1(ts) allele for 899 Dynein Heavy Chain, and treatment with $2 \%$ DMSO vehicle media or $2 \%$ DMSO + nocodazole media. Measurements at the permissive and restrictive temperature are differentiated by blue and red

901 coloring, respectively. Error bars are SEM. E) Representative $\mathrm{P}_{\text {sys-1 }}$ VENUS::SYS-1 and mCh::g-Tubulin for 902 each treatment in $\mathrm{D}$, respectively.

Figure 4: 
lacZ(RNAi) and ecps-1(RNAi) representative centrosomes in grayscale and intensity dependent LUT. C)

Putative dynein and proteasome adaptor ECPS-1 also appears to also enhance centrosomal ZYG-1

2(RNAi)) and D) Representative GFP::ZYG-1c centrosomal images in grayscale and intensity dependent

\section{Figure 5:}

\section{Effect of Dynein Depletion on nuclear SYS-1 Localization and Gene Expression in EMS Descendant}

918 Cells. A) The EMS precursor (embryo ventral cell) divides, receiving Wnt and Src specification and anterior, unsignaled MS daughter cells. Wildtype specification results in depicted approximate GFP::SYS-

9211 localization pattern specifically enriched in the signaled daughter nucleus. B) Representative images of

922 wildtype and ecps-1(RNAi) treated embryos. Orange box encloses the unsignaled MS nucleus, Blue box

923 encloses the signaled E nucleus. C) Quantification of GFP::SYS-1 nuclear enrichment in indicated dynein

924 RNAi knockdowns. C') Mean difference in nuclear GFP::SYS-1 enrichment from quantification in C. Error

926 lineage in the first (orange) second (blue) and third (green) lineage after the founding of the lineage (the

9272 2, 4, and 8 cell E descendants, respectively). D') Representative embryos exhibiting WT (all lacZ(RNAi)- 
Figure 6: one-sided Mann-Whitney test

Figure 7:

943 (bottom) SCs. Likely ectopic SC exhibit spatially distinct 'doublets', highlighted here in brackets. C)

944 Number and relative frequency of SCs via anatomical marker when in untreated adults and in

945 backgrounds with RNAi depletion of various dynein subunits. D) Number and relative frequency of SCs

946 via anatomical marker when SYS-1 is overexpressed via a heat shock-driven transgene in both untreated

947 larvae and in backgrounds with RNAi depletion of various dynein subunits. ${ }^{*}=p<0.05, * *=p<0.01, * * *=$

$948 \quad \mathrm{p}<0.001$ by Mann-Whitney test

\section{Figure 8:}


950 Proposed model. Speculation into the various processes by which SYS-1 and its regulators appear to be

951 localized to mitotic centrosomes. Both SYS-1 and some component of its centrosomal proteasome-

952 dependent negative regulators, here represented by purple circle, demonstrate some dependence on

953 microtubule mediated trafficking. While various dynein subunits are requisite components of a

954 functional dynein complex, the negative effect of their depletion on SYS-1 centrosomal steady state

955 indicates a preferential or more frequent trafficking of SYS-1. Relatively rare proteasome trafficking

956 events mediated by ECPS-1 meanwhile, are sufficient to maintain appropriate SYS-1 centrosomal

957 enrichment. This less efficient proteasomal trafficking, however, renders it vulnerable to Nocodazole-

958 mediated decrease in overall microtubule trafficking/network formation efficiency. Red inset depicts

959 decreased processivity or cargo binding capacity induced by dynein light chain RNAi depletions. Blue

960 inset depicts depletion of adapter ECPS-1 and resulting depletion of proteasome-trafficking specific

961 dynein complexes, while not otherwise affecting the microtubule motor. 


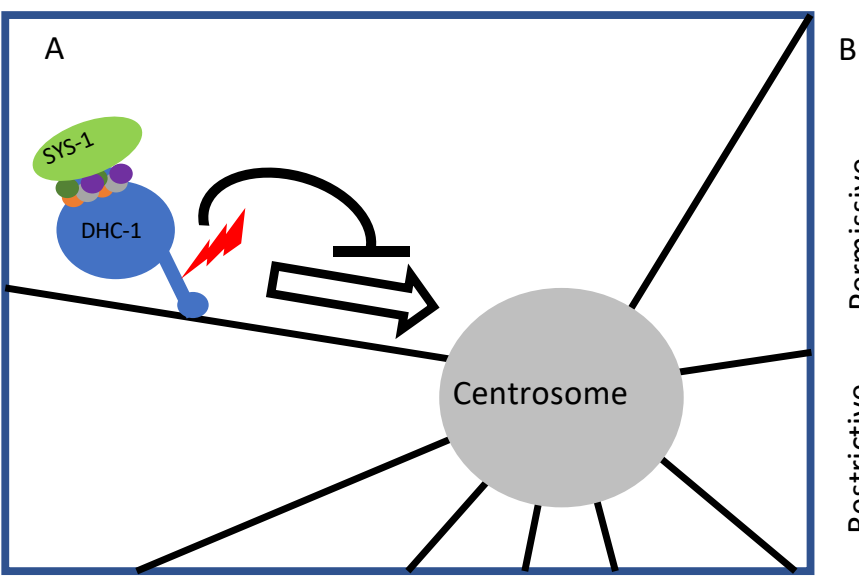

C

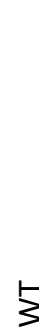
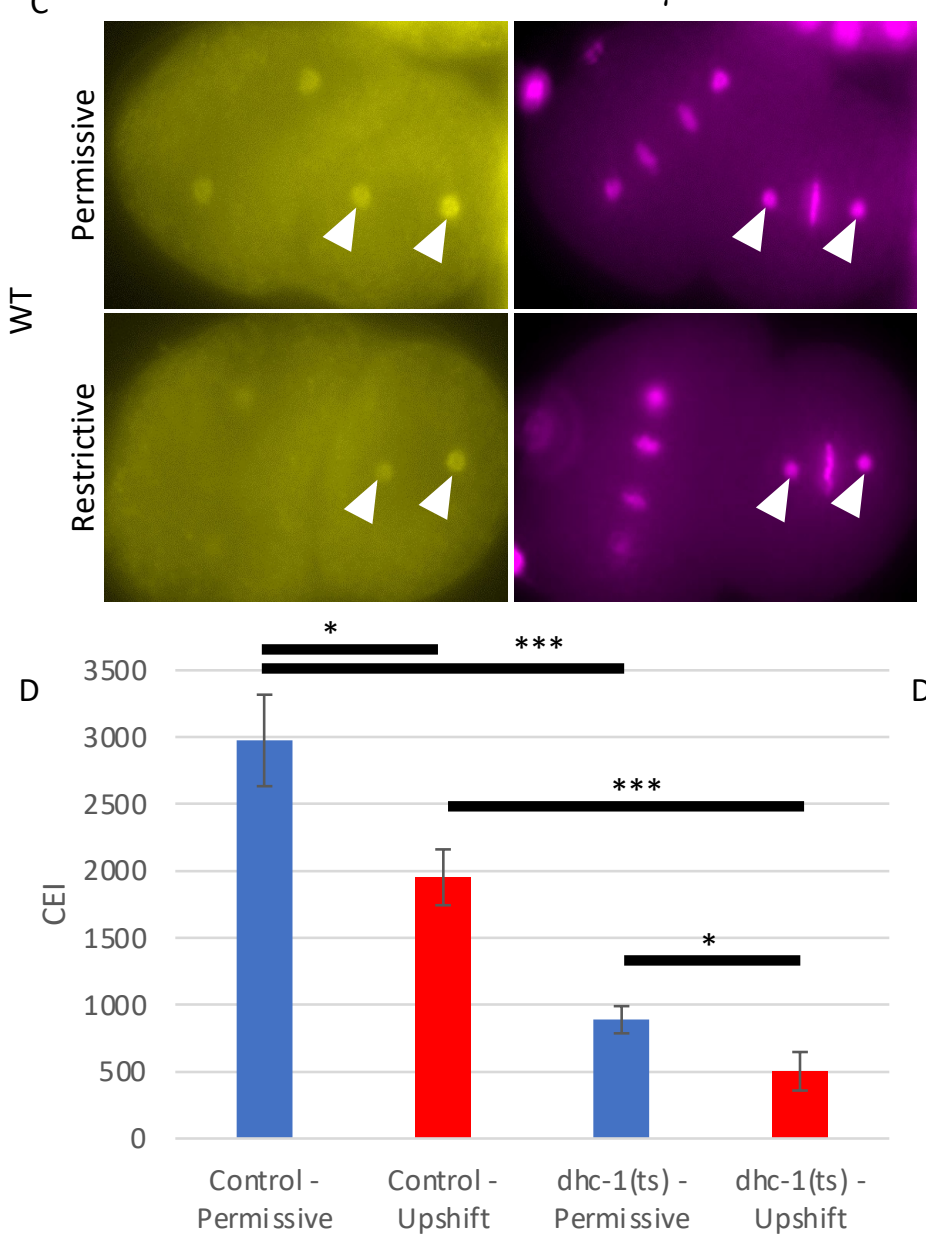

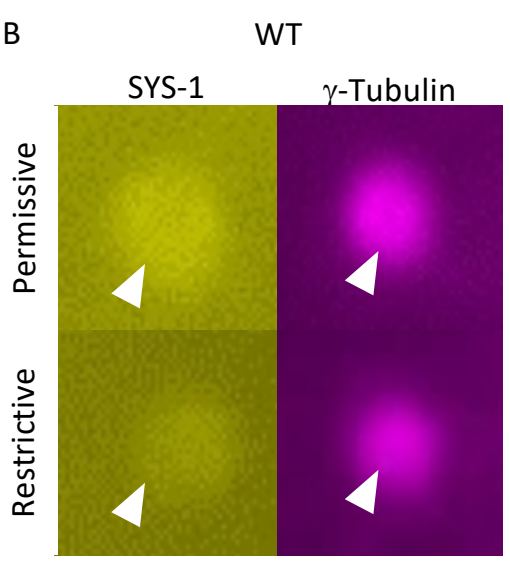

SYS-1

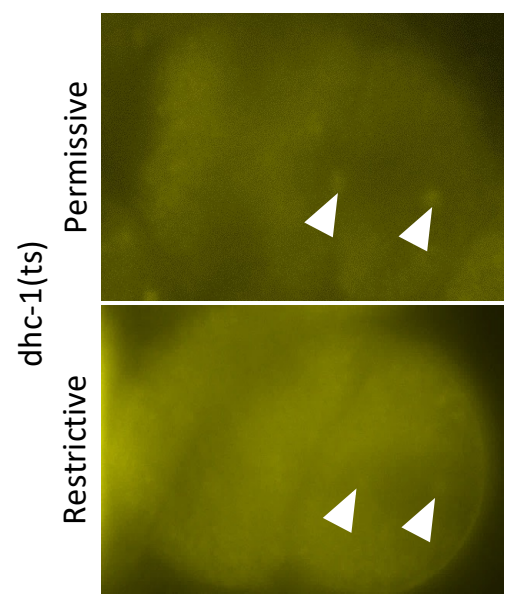

$D^{\prime}$

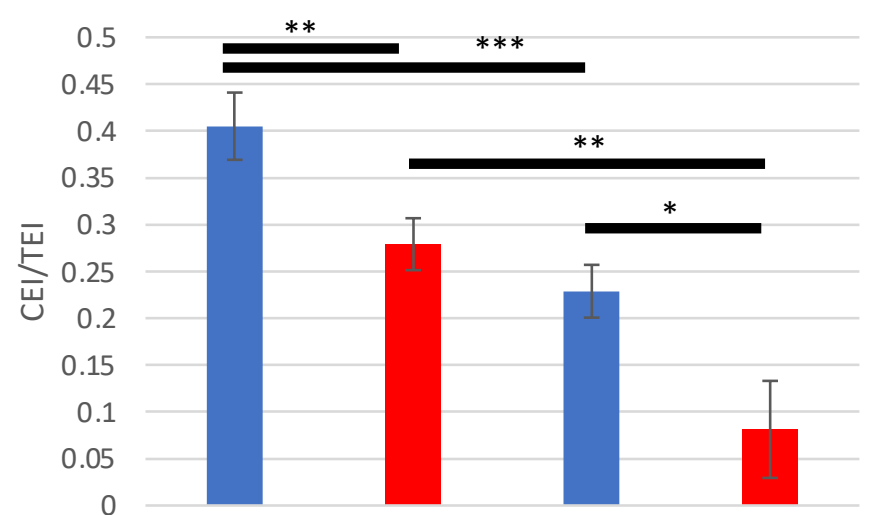

Control Permissive dhc-1(ts)

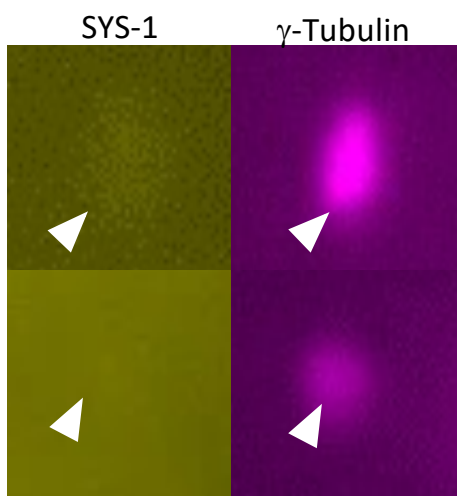

$\gamma$-Tubulin

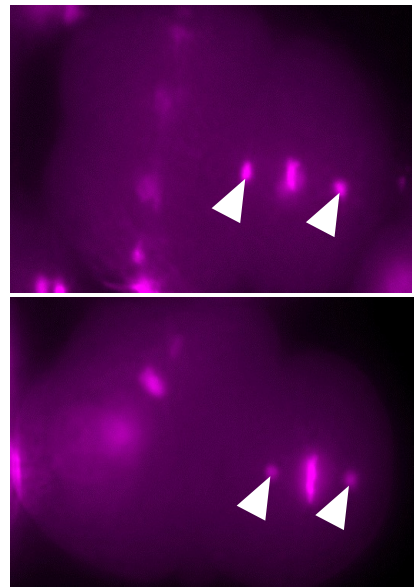

Control - dhc-1(ts) - dhc-1(ts) Upshift Permissive Upshift 
A

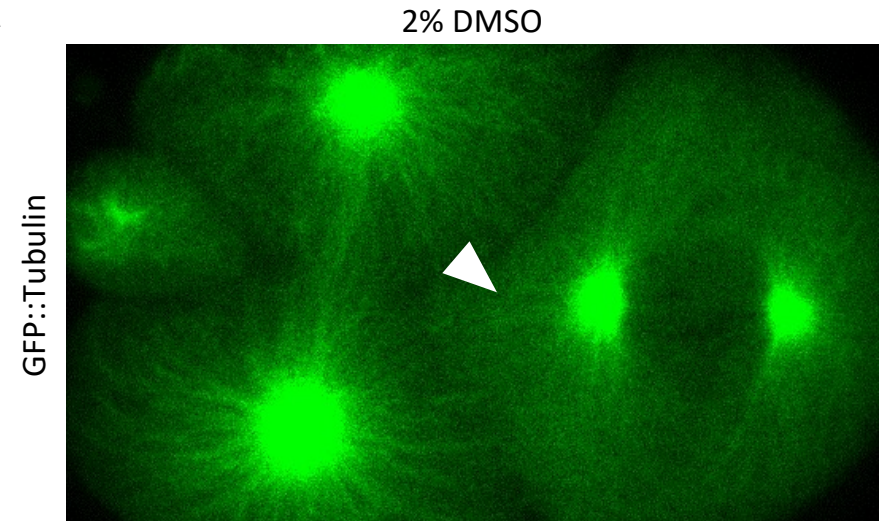

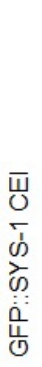

B
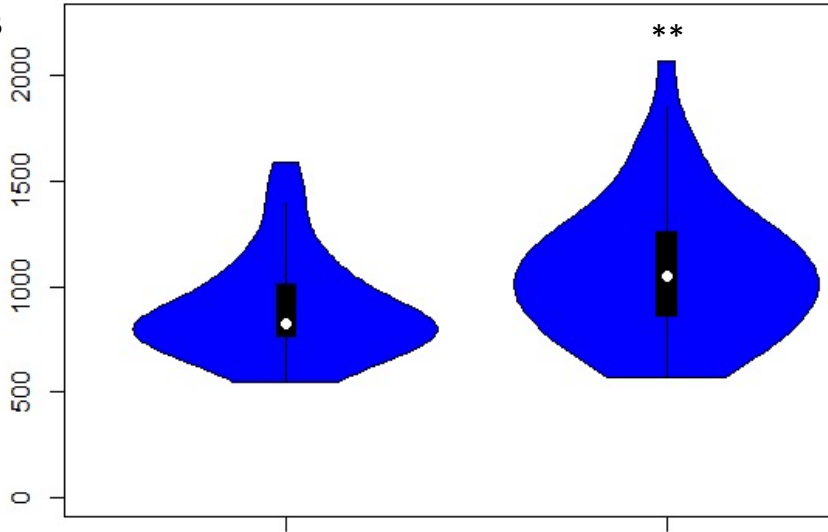

DMSO

D

D

0.25

$\underbrace{}_{\text {岀 }} 0.15$

0.1

0.05

0
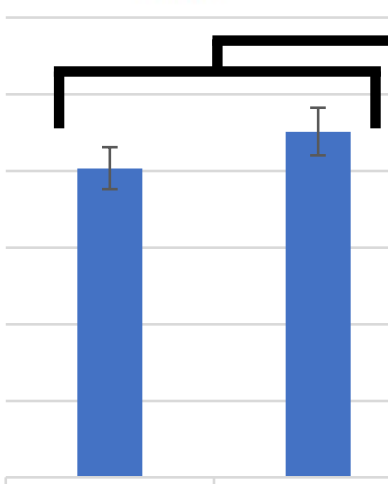

Permissive

DMSO

WT

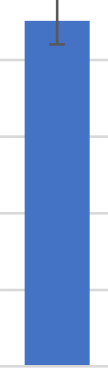

Permissive

nocodazole

WT
Nocodazole

$* *$
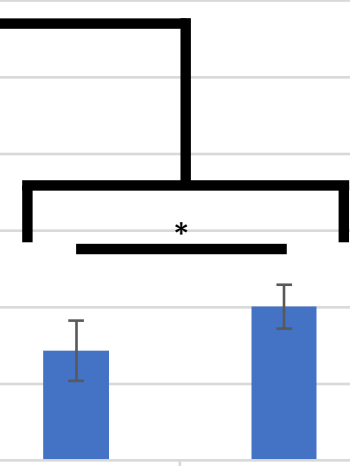

Permissive

DMSO

dhc-1(ts)
C

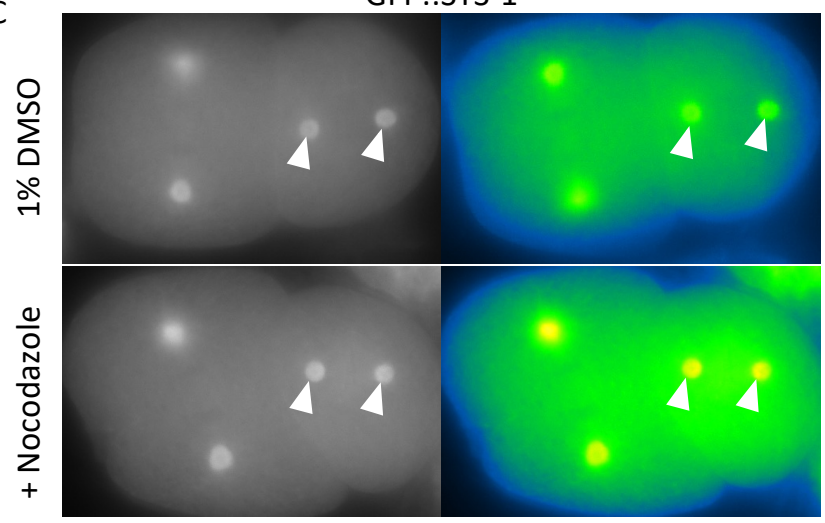

$25 \mu \mathrm{g} / \mathrm{ml}$ Nocodazole

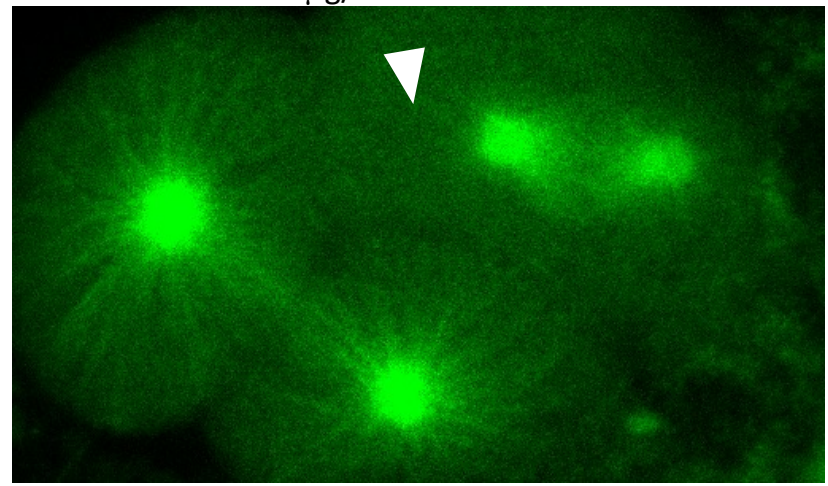

GFP::SYS-1

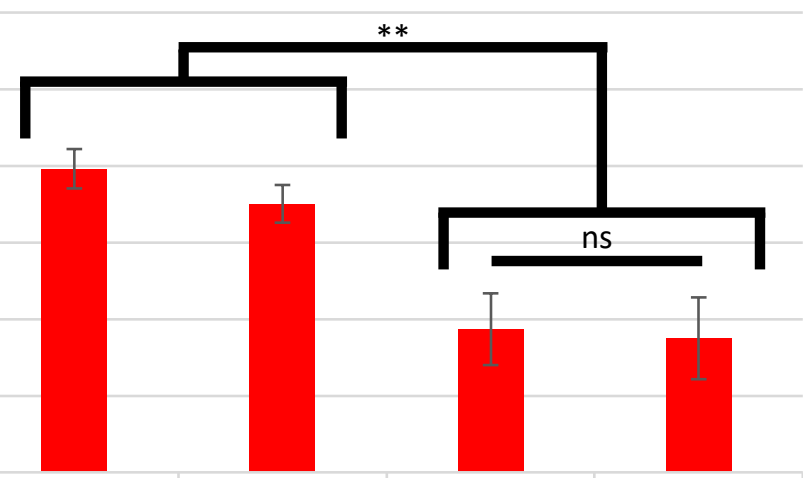

Restrictive

DMSO

Restrictive

nocodazole
Restrictive

DMSO

dhc-1(ts)

Restrictive

nocodazole

WT

dhc-1(ts)

E

sys-1::VNS::SYS-1

mCh:: $\gamma$-Tubulin 


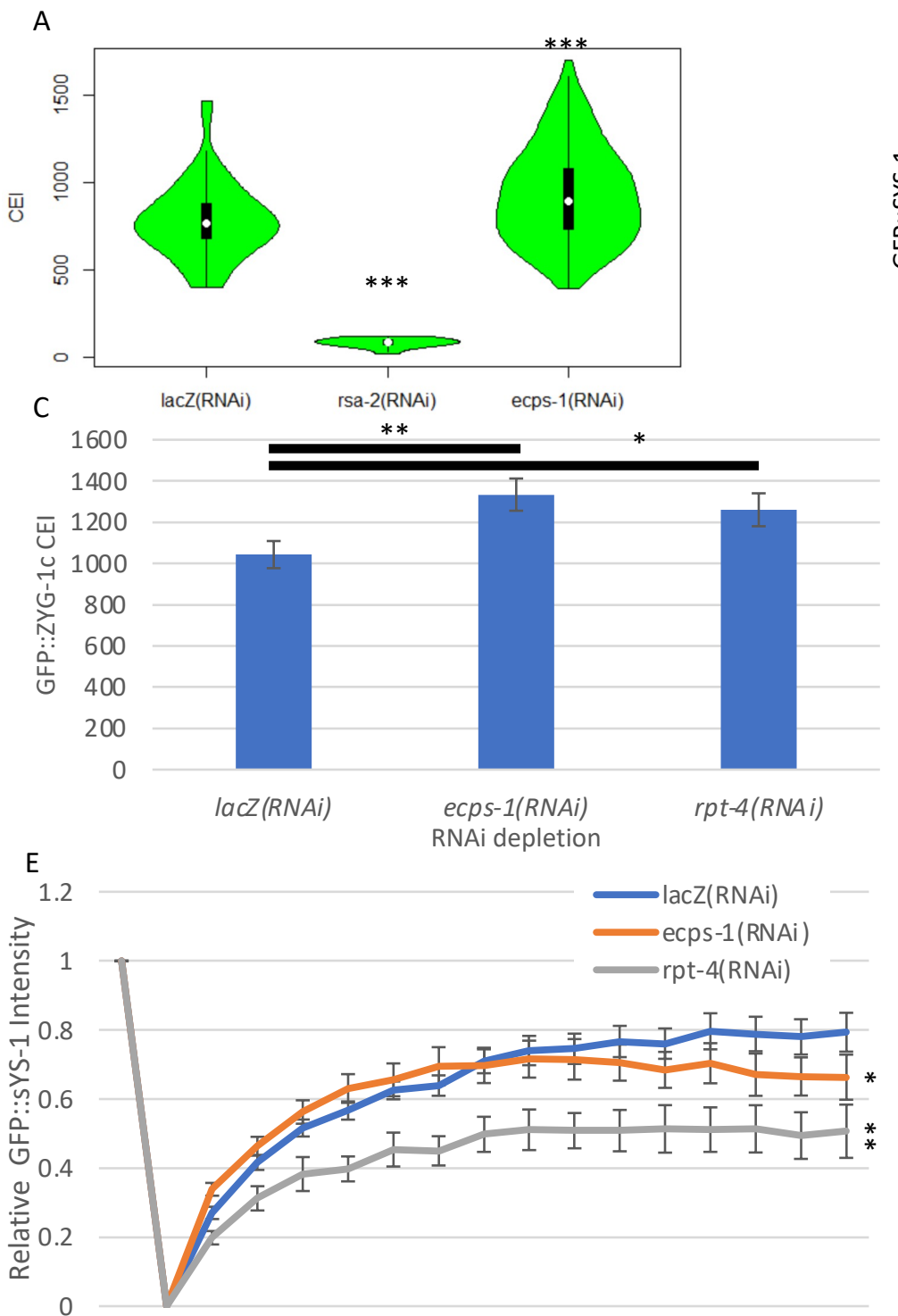

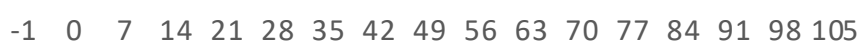
Time (S)
B

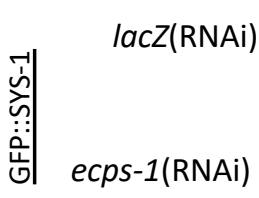

D
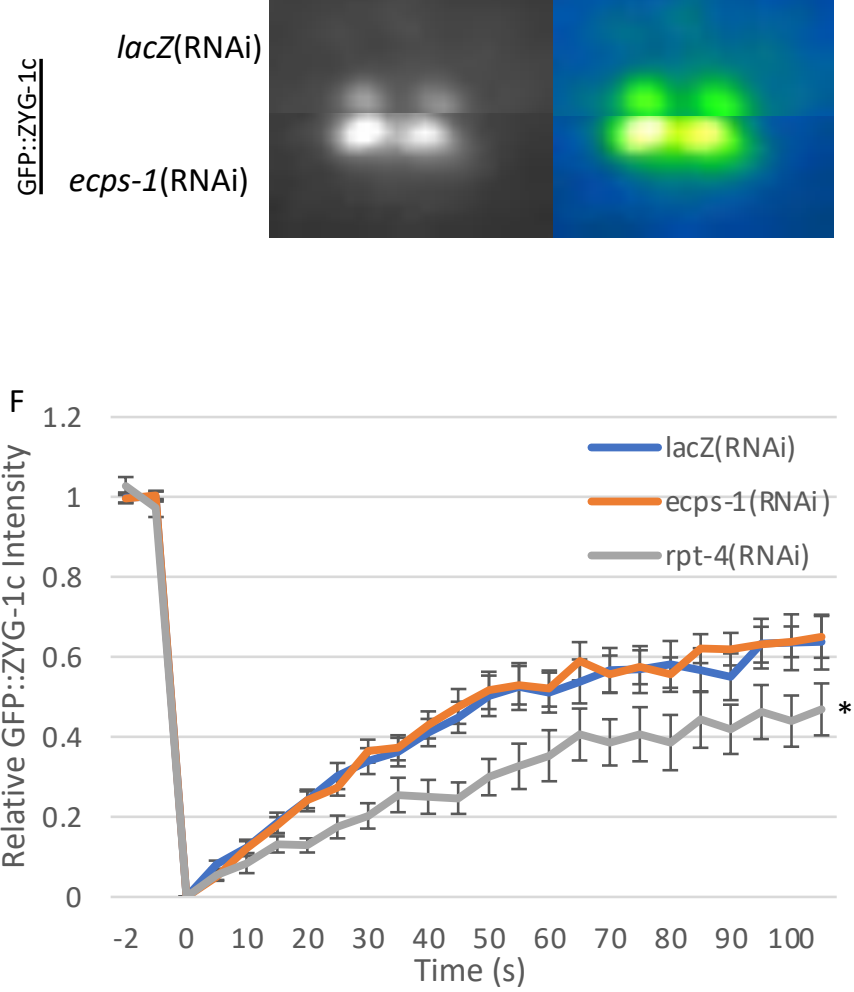

G - GFP::SYS-1 recovery in lacZ(RNAi)

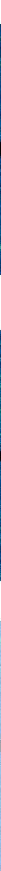


A

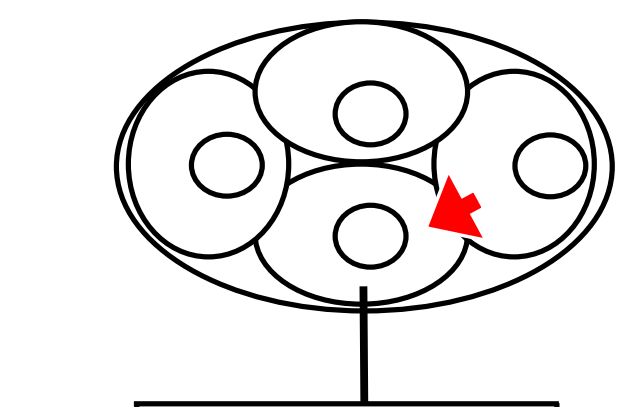

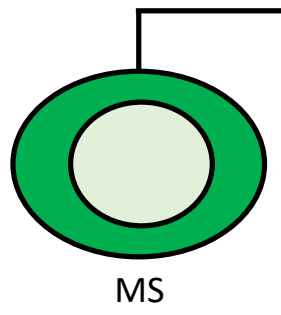

(Unsignaled)

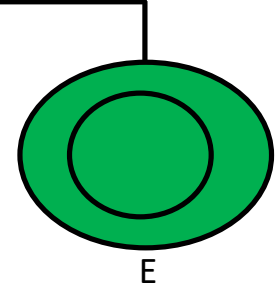

(Signaled)
B

$\operatorname{lacZ}(R N A i)$ ecps-1(RNAi)

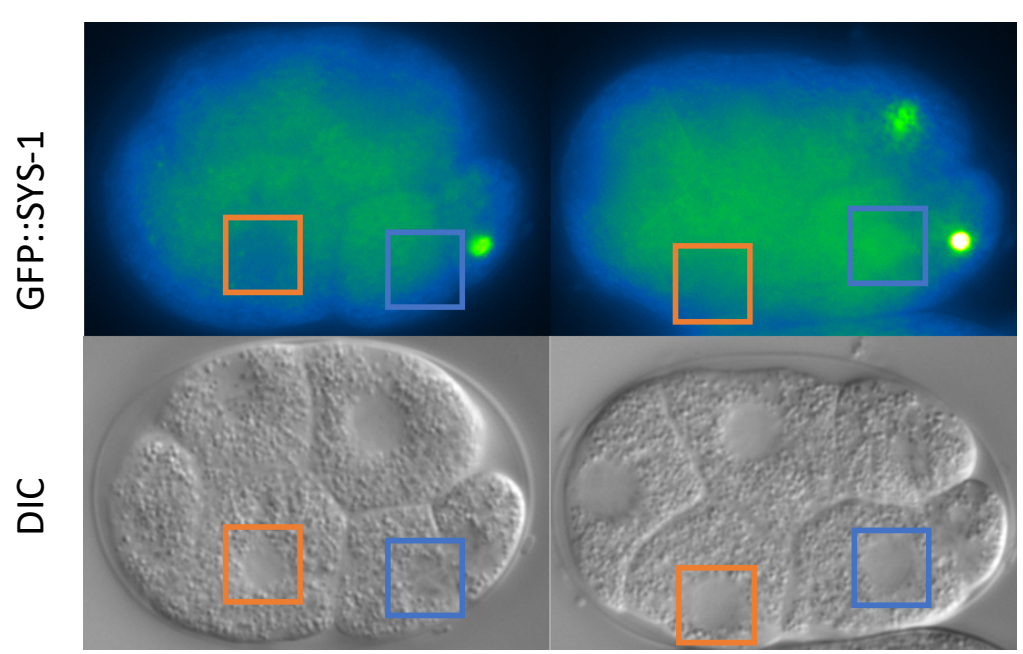

c
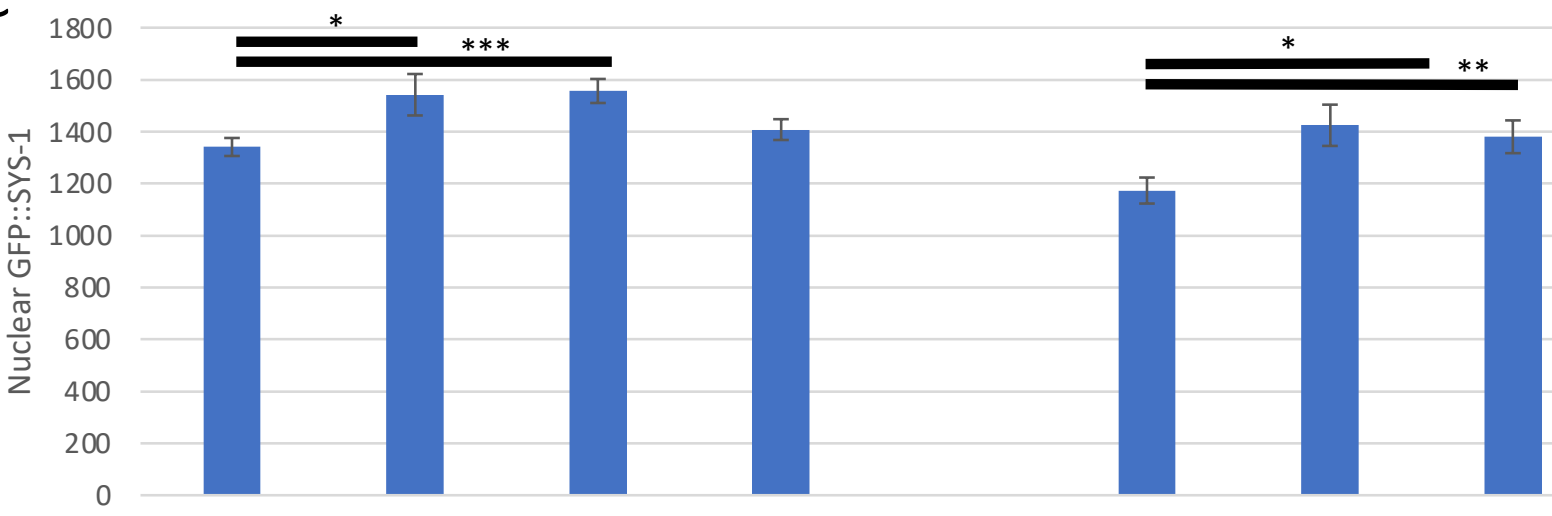

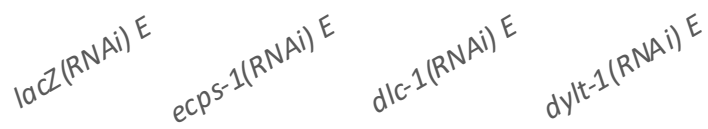

D

E descendants

D'
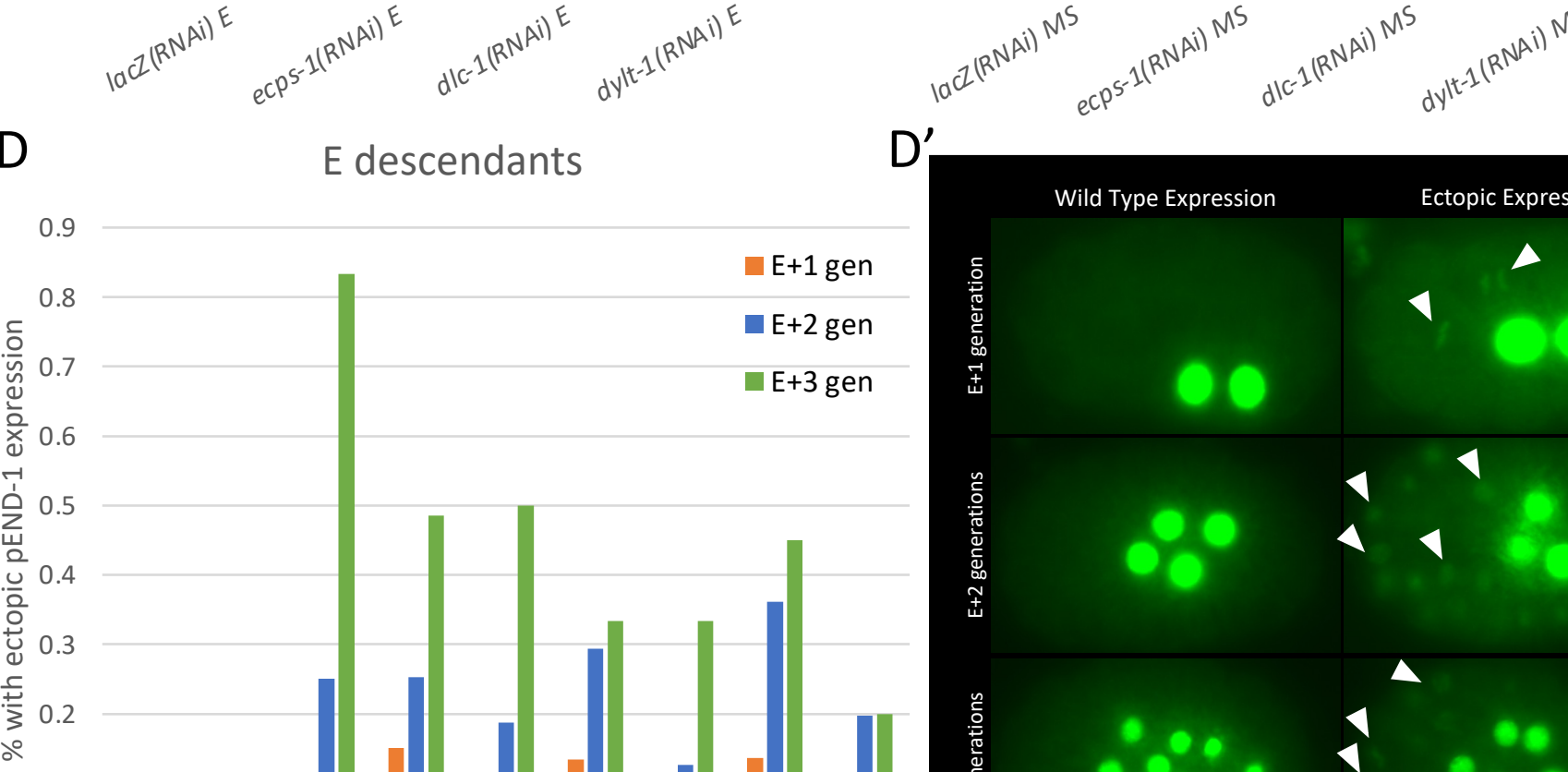

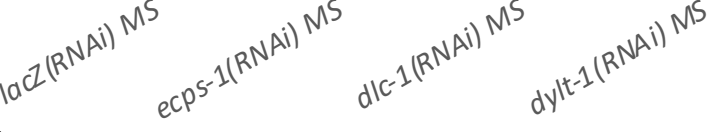

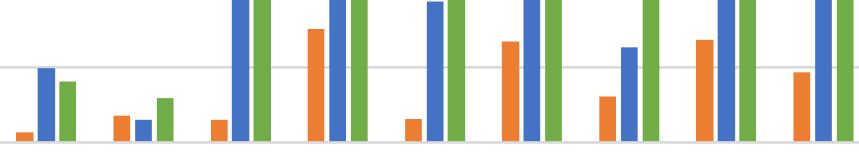

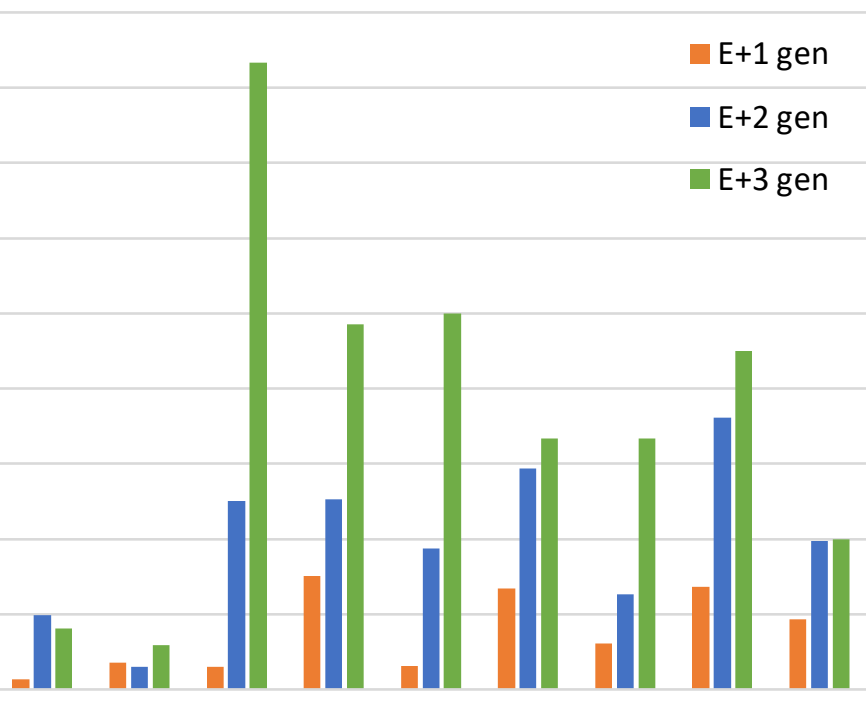

Wild Type Expression

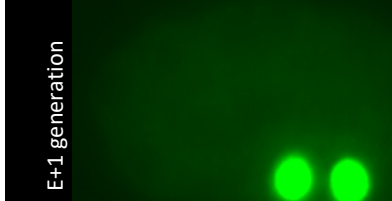

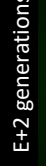

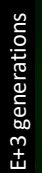

Ectopic Expression

$\Delta$

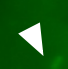

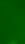
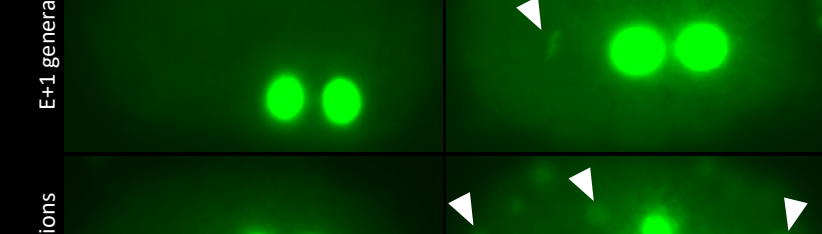

lacZ sys-1 dhc-1 dlc-1 dnc-1 dylt-1 ecps-1 rpt-4 rsa-2 RNAi treatment 
A
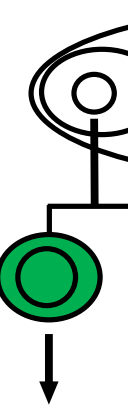

DTC
(Signaled)

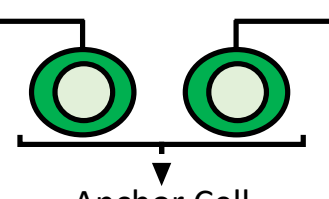

Anchor Cell

(Unsignaled)

C

$P_{h s}$-sys-1 (no hs); lacz(RNAi)

$P_{h s}$-sys-1 (no hs) sys-1(RNAi)

$P_{h s}-s y s-1$ (no hs) dhc-1(RNAi)

$P_{h s}-s y s-1$ (no hs) dlc-1(RNAi)

$P_{h s}$-sys-1 (no hs) dylt-1(RNAi)

$P_{h s}$-sys-1 (no hs) rsa-2(RNAi)

$P_{h s}-s y s-1$ (no hs); ecps-1(RNAi)
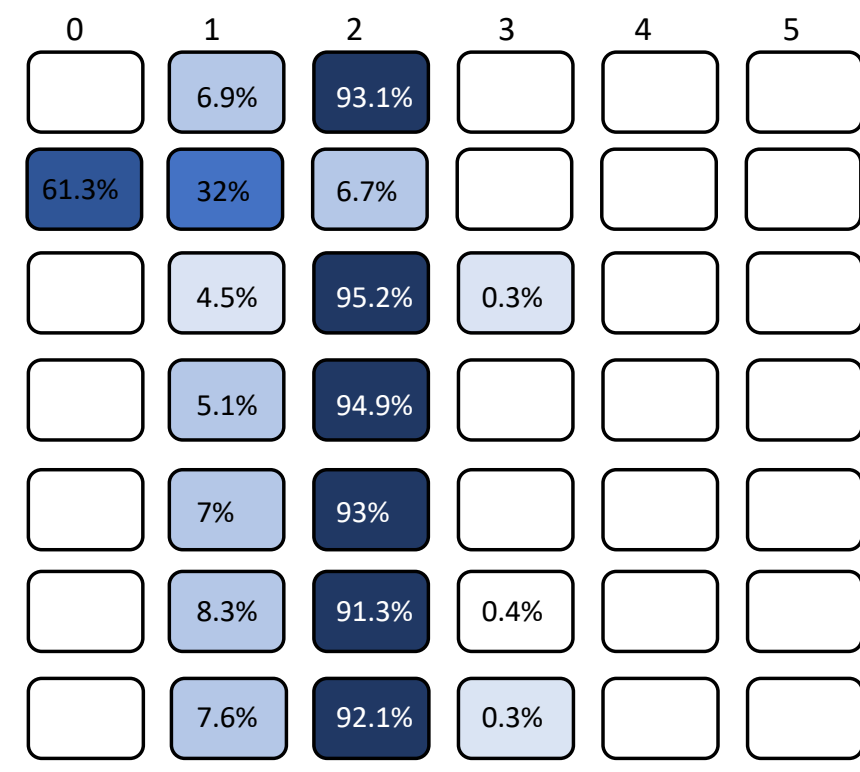

$95.2 \%$
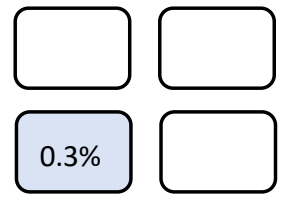

$94.9 \%$
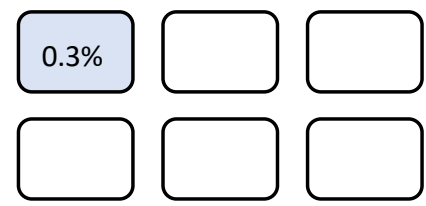

\section{$93 \%$}
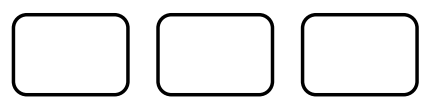

$91.3 \%$
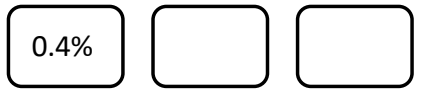

$92.1 \%$

4
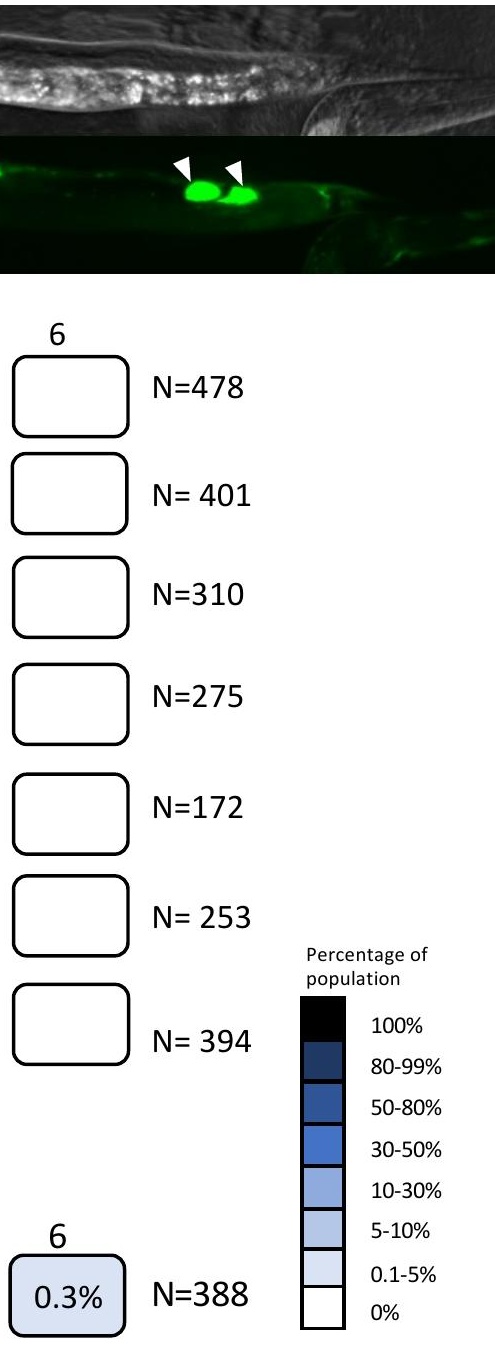

$N=394$

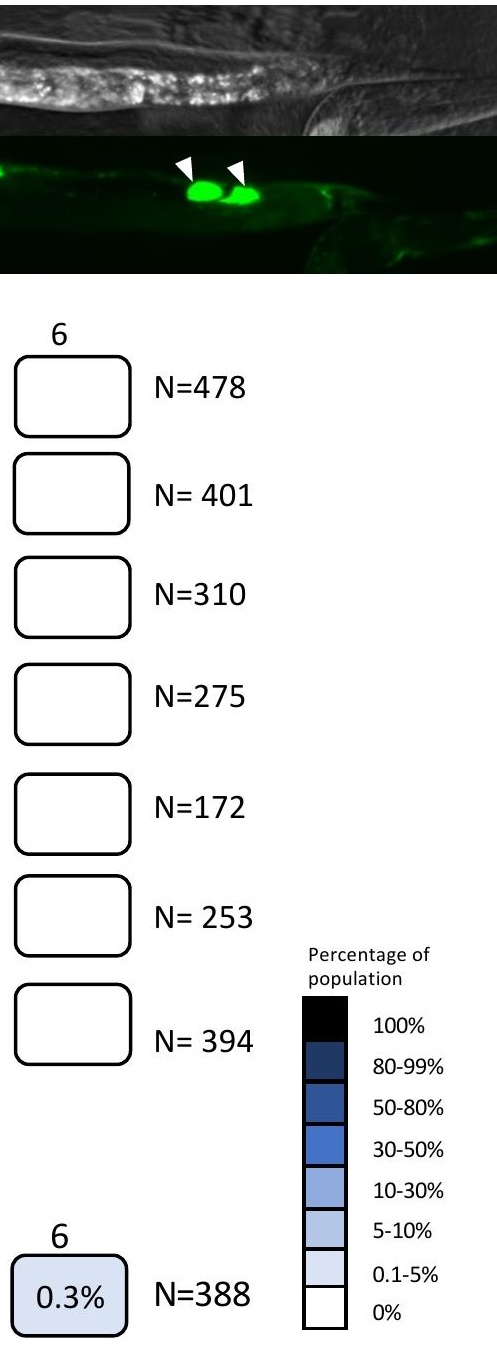
population

$100 \%$

$80-99 \%$

$50-80 \%$

$30-50 \%$

$10-30 \%$

5-10\%

$0.1-5 \%$

$69 \%$
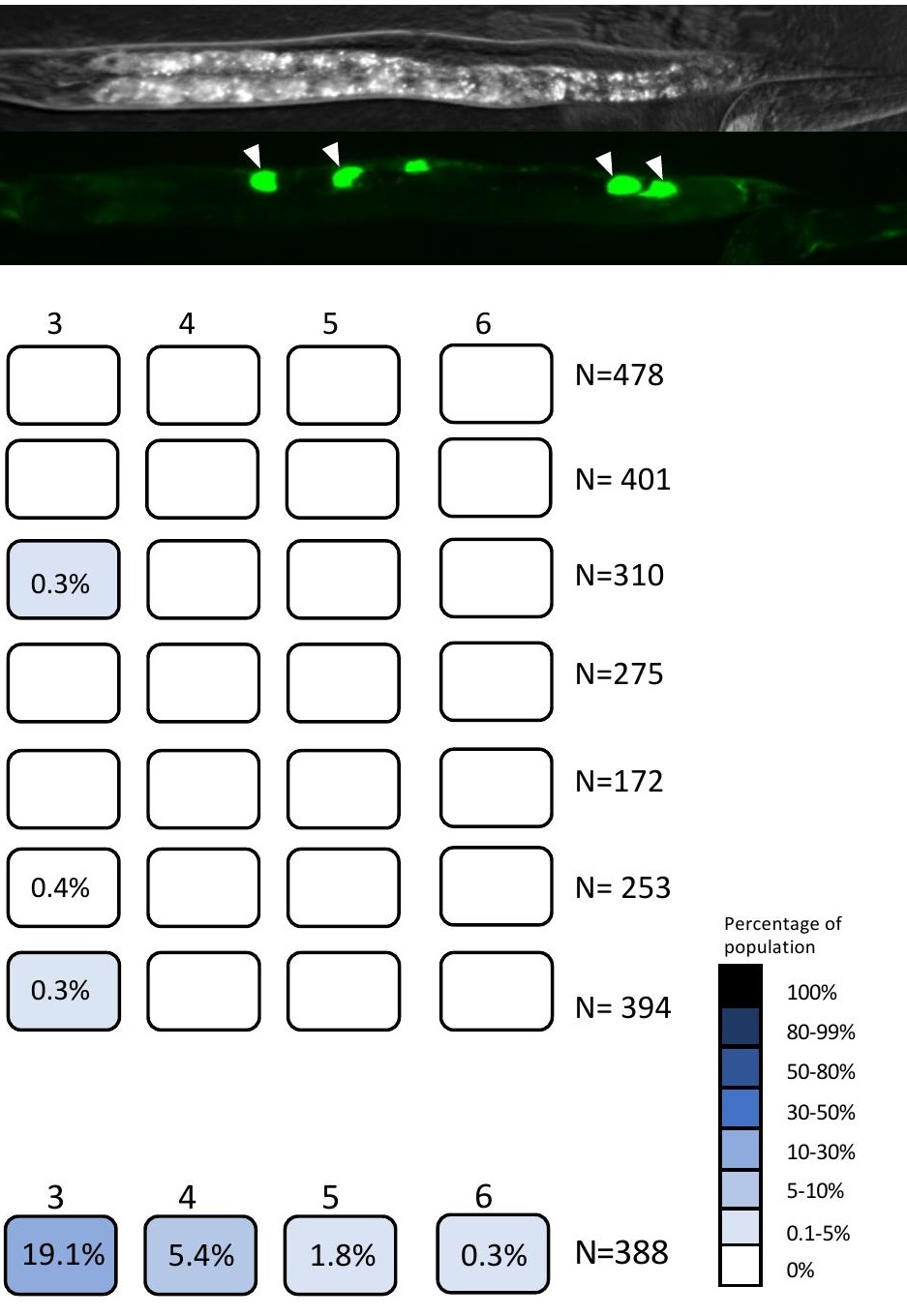

$0 \%$
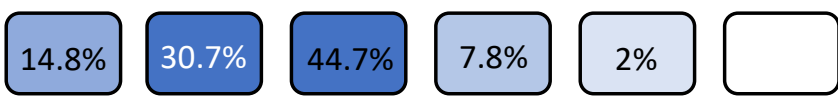

$P_{h s}-s y s-1(w / h s)$ sys-1(RNAi)

$P_{h s}-s y s-1(w / h s) d h c-1$ (RNAi)

$4.3 \%$

$58.1 \%$
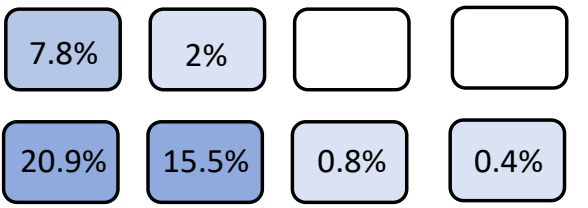

$N=358$

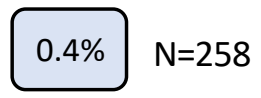

$71.9 \%$
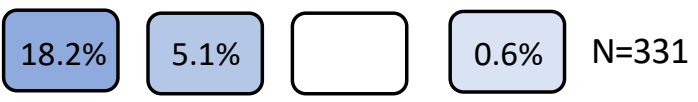

$P_{h s}-s y s-1(w / h s) d y / t-1(R N A i) * * *$

$6 \%$
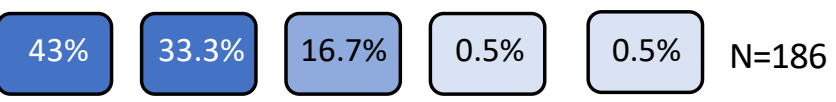

$62 \%$
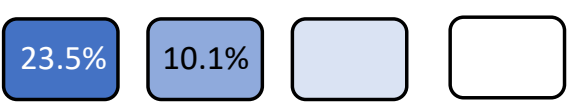

$N=323$

$64.4 \%$
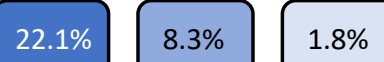

$0.3 \%$

$N=399$ 

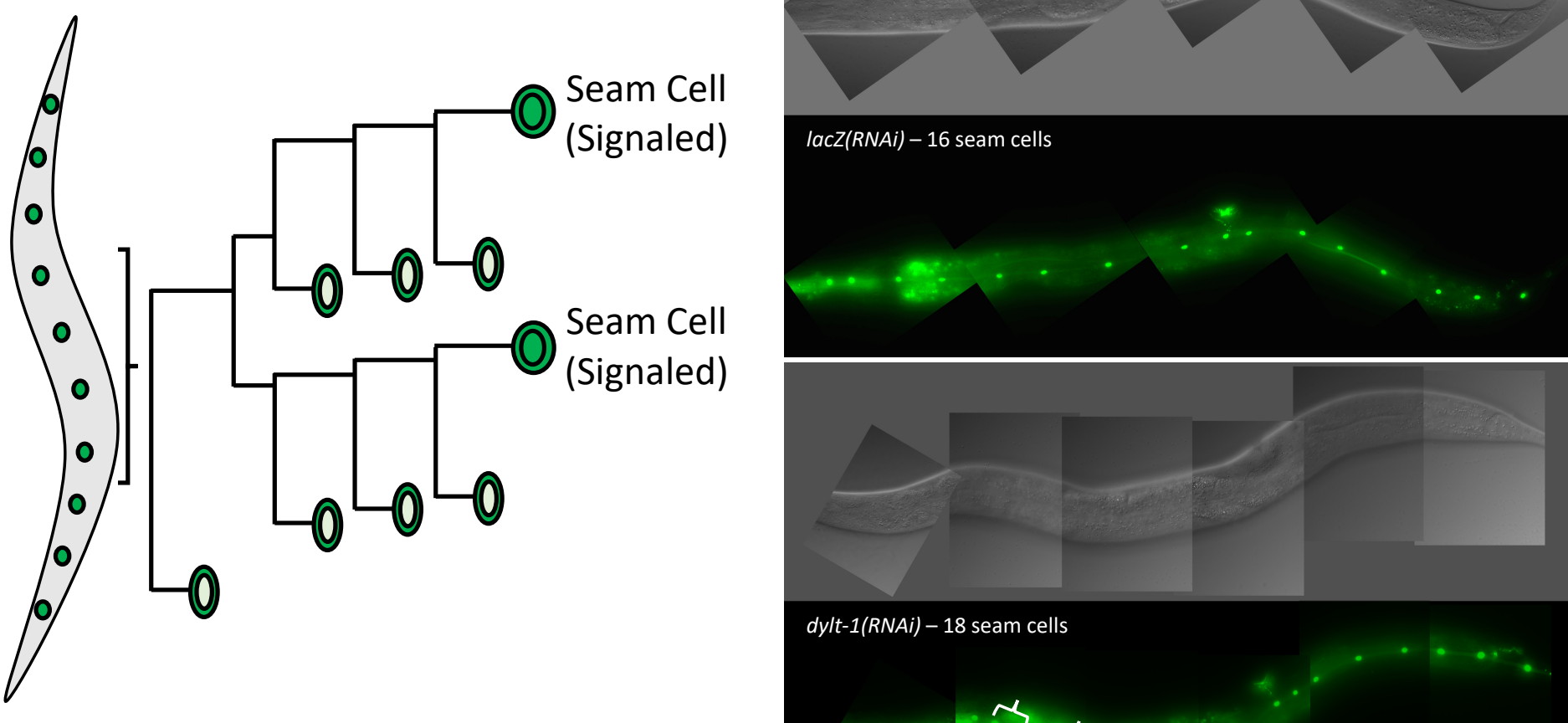

$d y / t-1(R N A i)-18$ seam cells

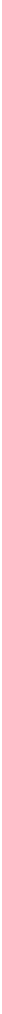




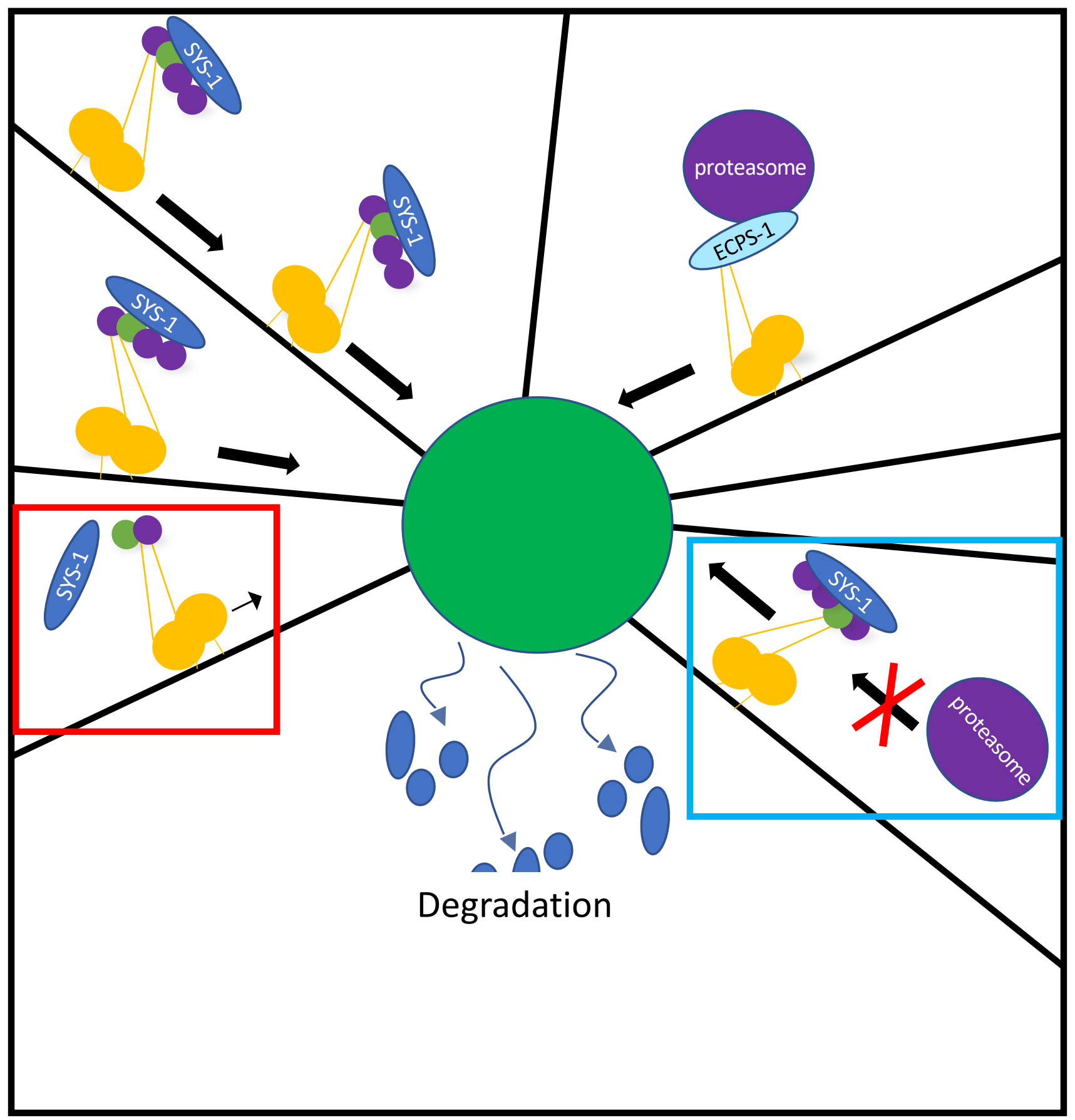

